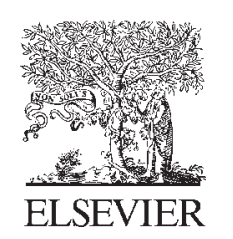

\title{
Recent advances in bioactive 1D and 2D carbon nanomaterials for biomedical applications
}

\author{
Ozlem Erol ${ }^{\mathrm{a}}$, Idil Uyan ${ }^{\mathrm{a}, \mathrm{c}}$, Meryem Hatip ${ }^{\mathrm{a}, \mathrm{b}}$, Canelif Yilmaz ${ }^{\mathrm{a}, \mathrm{c}}$, \\ Ayse B. Tekinay ${ }^{a, b, c}$, Mustafa O. Guler ${ }^{a, b, d, *}$ \\ ${ }^{a}$ National Nanotechnology Research Center (UNAM), Bilkent University, Ankara, Turkey \\ ${ }^{\mathrm{b}}$ Institute of Materials Science and Nanotechnology, Bilkent University, Ankara, Turkey \\ ${ }^{\mathrm{c}}$ Neuroscience Program, Bilkent University, Ankara, Turkey \\ ${ }^{\mathrm{d}}$ Institute for Molecular Engineering, University of Chicago, Chicago, IL, USA 60637 \\ Received 22 October 2016; accepted 6 March 2017
}

\begin{abstract}
One-dimensional (1D) carbon nanotubes (CNTs) and the two-dimensional (2D) graphene represent the most widely studied allotropes of carbon. Due to their unique structural, electrical, mechanical and optical properties, 1D and 2D carbon nanostructures are considered to be leading candidates for numerous applications in biomedical fields, including tissue engineering, drug delivery, bioimaging and biosensors. The biocompatibility and toxicity issues associated with these nanostructures have been a critical impediment for their use in biomedical applications. In this review, we present an overview of the various materials types, properties, functionalization strategies and characterization methods of $1 \mathrm{D}$ and $2 \mathrm{D}$ carbon nanomaterials and their derivatives in terms of their biomedical applications. In addition, we discuss various factors and mechanisms affecting their toxicity and biocompatibility.
\end{abstract}

(C) 2017 Elsevier Inc. All rights reserved.

Key words: Carbon nanotube; Graphene; Graphene oxide; Biomedical applications; Biocompatibility

After the discovery of zero-dimensional fullerenes, ${ }^{1}$ one dimensional (1D) carbon nanotubes $(\mathrm{CNT})^{2}$ and twodimensional (2D) graphene sheets ${ }^{3}$ were developed and found to possess excellent materials characteristics for a variety of applications. Increasing attention has been given to these "next-generation" materials during the past decade, and much effort has been expended to explore their unique properties and utilize them in fields ranging from energy storage to biomedicine. These materials have been widely used in biomedical applications and there is a growing and relevant concern for their toxicity and environmental effects, which remain modestly characterized.

The CNTs are hollow cylindrical sheets of hexagonal networks of carbon atoms. They exhibit very high aspect ratio, measuring a few nanometers in diameter and up to several microns in length. They can be metallic or semiconductive, depending on their chirality and add-atoms, and their electronic, mechanical and optical properties are ideal for a broad range of purposes. In addition, CNTs have large surface area, small diameter and high curvature, which allow them to effectively interact with biomolecules through van der Waals, $\pi-\pi$ stacking and hydrophobic interactions. These properties can also be used to facilitate the surface modification of CNTs in order to increase their solubility is aqueous media or modulate the covalent attachment of functional groups for biomedical applications. ${ }^{4}$ The biological utility of CNTs is further improved by their optical absorption in the NIR-IR window and fluorescence emission in the NIR-II window, which makes them well-suited for photothermal therapy, photoacoustic imaging and deep-tissue fluorescence imaging. ${ }^{5}$ Due to their fibrous structure and strong mechanical integrity, CNTs can also be used as a reinforcing material in tissue engineering applications and provide electrical conductivity in regenerative scaffolds to direct cellular growth and differentiation. ${ }^{6}$

Conflict of interest: The authors declare no conflicts of interest.

*Corresponding author at: Institute for Molecular Engineering, University of Chicago, Chicago, IL, USA 60637.

E-mail address: mguler@uchicago.edu (M.O. Guler). 
Graphene is a single layer of honeycomb carbon lattice and the basic building block of all graphitic forms. Similar to CNTs, graphene and its derivatives demonstrate unique mechanical, electrochemical and optical properties and interact with biomolecules through $\pi-\pi$ stacking and/or electrostatic interactions, which are of great value for drug loading and biosensor design applications. In addition, the rich oxygen-containing groups that are attached to graphene oxide (GO) can be directly functionalized by biological ligands to facilitate targeted imaging and drug delivery. The high and intrinsic near-infrared (NIR) absorbance of GO allows it to be used as photo-thermal agents for cancer treatment with strong therapeutic outcomes. ${ }^{7}$

In this review, we present biomedical applications of CNT, graphene and GO based materials as representatives of 1D and 2D carbon nanostructures. We describe the structure, type, distinguishing properties, synthesis and purification methods, characterization techniques, and functionalization of CNTs, graphene and their derivatives. Then, their biocompatibility is discussed in terms of their cytotoxicity, genotoxicity and inflammatory responses elicited by these nanostructures. Lastly, their biomedical applications, including their use in tissue engineering, drug delivery, bioimaging and biosensors, are presented with examples from the recent works.

\section{D and 2D carbon nanomaterials}

\section{Carbon nanotubes}

CNTs are one-dimensional, hollow, tubular, nanostructured allotropes of carbon. They are composed of one or more layers of graphene sheet(s) that are rolled into a seamless cylinder, with at least one end typically capped with hemisphere of buckyball. CNTs have attracted great attention in various applications; including energy storage, ${ }^{8}$ polymer reinforcement, ${ }^{9}$ sensors, ${ }^{10}$ photonics, ${ }^{11}$ catalysis ${ }^{12}$ and recently biomedical engineering ${ }^{13}$ because of their unique and unusual structural, electrical, mechanical, thermal, and optical properties.

\section{Structure and properties of carbon nanotubes}

The discovery of CNTs dates back to the 1960s, with the identification of graphite whiskers in the form of rolled-up sheets of graphite layers by Bacon. ${ }^{14}$ This was followed by the discovery of fullerenes, which are singular sheets of graphene that are curved spherically to form closed cages, in $1985 .{ }^{1}$ Iijima subsequently demonstrated the production of multi-walled carbon nanotubes (MWCNTs) in 1991, using a method similar to that used for fullerene synthesis. ${ }^{2}$

The backbone of CNT is built from the hexagonal carbon bonds of $\mathrm{sp}^{2}$-hybridized carbon atoms, which are arranged into hollow, cylindrical nanostructures. Although CNTs have similar hexagons of $\mathrm{sp}^{2}$ hybridized $\mathrm{C}-\mathrm{C}$ bonds like graphene, they possess distinctive properties because of their high aspect ratios, large surface areas, ultra-small diameters and non-planar nature. When a graphene layer bends to form CNTs, $\sigma$-bonds that lie in the $\mathrm{sp}^{2}$ plane are shifted out of plane and reside outside the curvature of the nanotube sidewall, which causes $\pi$-orbitals to be more delocalized outside the nanotube, inducing electron cloud distortion and resulting in a rich $\pi$-electron conjugation outside the tubular structure. ${ }^{15}$ This structural difference imparts CNTs with unusual electrochemical and thermal properties as well as high mechanical strength.

The CNTs can be classified as single or multi-walled. The outer diameter of single-walled CNTs (SWCNTs) varies between $0.4 \mathrm{~nm}$ to $2 \mathrm{~nm}$, while that of multi-walled CNTs (MWCNTs) ranges from 2 to $100 \mathrm{~nm}$, depending on the synthesis conditions. ${ }^{16}$ Both SWCNTs and MWCNTs can reach lengths of 0.2 to several $\mathrm{mm}$, resulting in very high $(>10000)$ aspect ratio in many cases. Chirality (i.e., the angle between the $\mathrm{C}-\mathrm{C}$ bonds and the nanotube axis) is another essential parameter for the structure and properties of CNTs. Representation of chiral, achiral zig-zag and armchair structures are shown in Figure 1. This classification can be used to determine whether a particular SWCNT arrangement is semiconductive or metallic. ${ }^{17}$ On the other hand, the chirality of each wall is different in the case of MWCNTs, which exhibit metallic character. Electrical conductivity can reach to $10^{6} \mathrm{Scm}^{-1}$ for SWCNTs and $10^{4} \mathrm{Scm}^{-1}$ for MWCNTs.

A one-dimensional tubular morphology and $\mathrm{sp}^{2}$ hybridized $\mathrm{C}-\mathrm{C}$ bonds (which are stronger than $\mathrm{sp}$ and $\mathrm{sp}^{3}$ bonds) also provide CNTs with unique mechanical properties, such as Young's moduli $(\sim 1 \mathrm{TPa})$ and tensile strengths $(\sim 50 \mathrm{GPa})$ that are much higher than those of steel. ${ }^{19}$ In addition, CNTs have lower density compared to many high-strength materials. These good mechanical characteristics, in tandem with their light weight and flexibility, make CNTs effective candidates for reinforcing composites. CNTs also have excellent thermal conductivity and stability, which are important issues in the electronics industry to prevent structural damage. ${ }^{20}$

In general, large-scale production of MWCNTs is relatively easier compared to SWCNTs, which enhances the former's popularity for biomedical applications. Although MWCNTs are more stable than SWCNTs, they are also more inert and less soluble in aqueous media. The CNTs tend to entangle and form bundles or crystalline ropes due to their high aspect ratio and strong van der Waals (more specifically $\pi-\pi$ stacking) interactions between them. ${ }^{21}$ The CNTs also exhibit strong optical absorption in NIR-IR window (750-1000 nm), enabling their use in photothermal therapy and photoacoustic imaging. SWCNTs are particularly suitable in deep-tissue fluorescence imaging as fluorescence contrast agents due to their intrinsic fluorescence emission in the NIR-II window $(1000-1700 \mathrm{~nm}) .^{5}$

\section{Synthesis of carbon nanotubes}

The method used to synthesize CNTs is a very important part of CNT research, because the diameter, length, morphology, structure, chirality, quality and purity of the resulting structure depend strongly on the preparation method. Arc-discharge method is one of the most widely used techniques to obtain CNTs. In this method, an electric discharge is produced between two graphite electrodes in an inert atmosphere. This electric discharge produces a high temperature (around $3000{ }^{\circ} \mathrm{C}$ ), resulting in the evaporation of a carbon electrode and its subsequent deposition on the other electrode. If the graphite electrode contains metal catalysts such as cobalt, nickel or iron, SWCNTs are formed; while a lack of metal catalysts produces MWCNTs. ${ }^{22}$ The diameter of the CNTs can be controlled by 

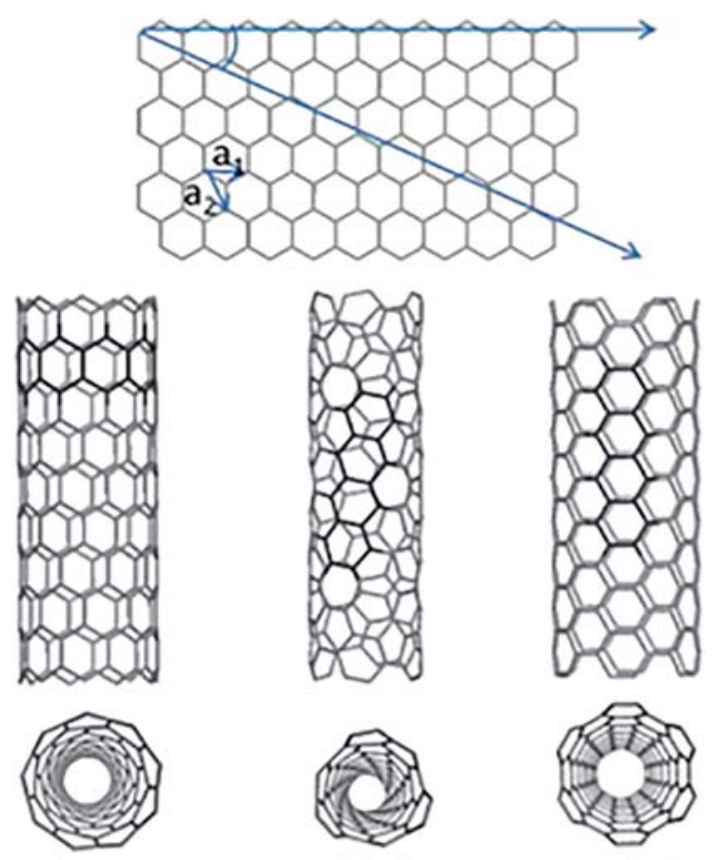

zigzag

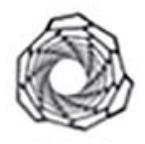

chiral

\section{Purification of carbon nanotubes}

In all above-mentioned synthesis methods, CNTs contain impurities that are mostly metallic catalyst particles, amorphous carbon, and carbonaceous fragments which alter the electrical and mechanical properties of CNTs. These impurities can also be detrimental for their biomedical use. Differences in aspect ratios, sizes, oxidation rate and solubility between impurities and CNTs can be utilized to eliminate the former and improve the material properties of the latter. Various purification techniques, such as liquid and gas phase oxidations, filtration, and microwave heating, have been developed for this purpose. ${ }^{29}$ It is essential to eliminate the impurities without destroying the tubular structure and intrinsic properties of CNTs. Oxidation is the most commonly used purification method, and includes liquid phase oxidation (such as acid treatment with $\mathrm{HNO}_{3}, \mathrm{HCl}, \mathrm{H}_{2} \mathrm{SO}_{4}$ etc. and/or refluxing in water or $\mathrm{H}_{2} \mathrm{O}_{2}$ ) and gas phase oxidation (heating in air, oxygen, or other gases). ${ }^{29,30}$ Liquid phase oxidation typically removes metal catalysts, amorphous carbon and some fullerenes by refluxing CNTs with acids. The disadvantages of this method are that it causes defects on the surface of the CNTs and introduces oxygenated functional groups such as carboxylic acids. In contrast, gas phase oxidation selectively removes the carbonaceous impurities by heating the $\mathrm{CNT}$ at a controlled rate to temperatures around $330{ }^{\circ} \mathrm{C}$. This method is unable to eliminate metal catalysts, which necessitate acid treatment such as $\mathrm{HCl}$ washing. In addition, as a chemical oxidative-based method, microwave heating treatment followed by treatment of $\mathrm{HCl}$ drastically reduces processing times to $\sim 1 \mathrm{~h}$ compared to conventional acid reflux methods (45h). ${ }^{31}$ Besides the chemical purification techniques, mass- or size-based purification methods such as microfiltration and chromatography are also used for the elimination of CNT impurities. These are relatively mild methods compared to their chemical counterparts. $^{29}$

\section{Graphene and graphene oxide}

Graphene is the first two-dimensional (2D) lattice of carbon atoms to be discovered, and consists of a peculiar honeycomb structure and layers that are one atom thick each. Graphene and its oxidized derivatives (GO or reduced graphene oxide (rGO)) uniquely combine properties such as high electronic and thermal conductivities, mechanical strength and impermeability to gases. ${ }^{32}$ While graphene could be cytotoxic, pharmaceutical and biomedical applications can benefit greatly from non-toxic, biocompatible and water-dispersible graphene layers that are produced through chemical functionalization with various ligands. ${ }^{33}$

\section{Structure and properties of graphene and graphene oxide}

Graphene family materials include few-layer graphene, graphene sheet, GO, and rGO sheet (Figure 2). ${ }^{34}$ Graphene consists of a single-layer sheet of trigonally bonded $\mathrm{sp}^{2}$ carbon atoms that is $0.35-1.6 \mathrm{~nm}$ in thickness ${ }^{33}$ and displays a compactly packed honeycomb crystal structure. ${ }^{35} \mathrm{GO}$, which is produced by the oxidation of graphite crystals, instead contains partly tetrahedrally bonded $\mathrm{sp}^{3}$ carbon atoms, which are located slightly above or below the graphene plane. Several observations confirm the presence of defective regions on GO surfaces, which interact readily with functional groups. According to Pandey et al., ${ }^{36}$ the random distribution of non-oxidized and oxidized areas with 
A
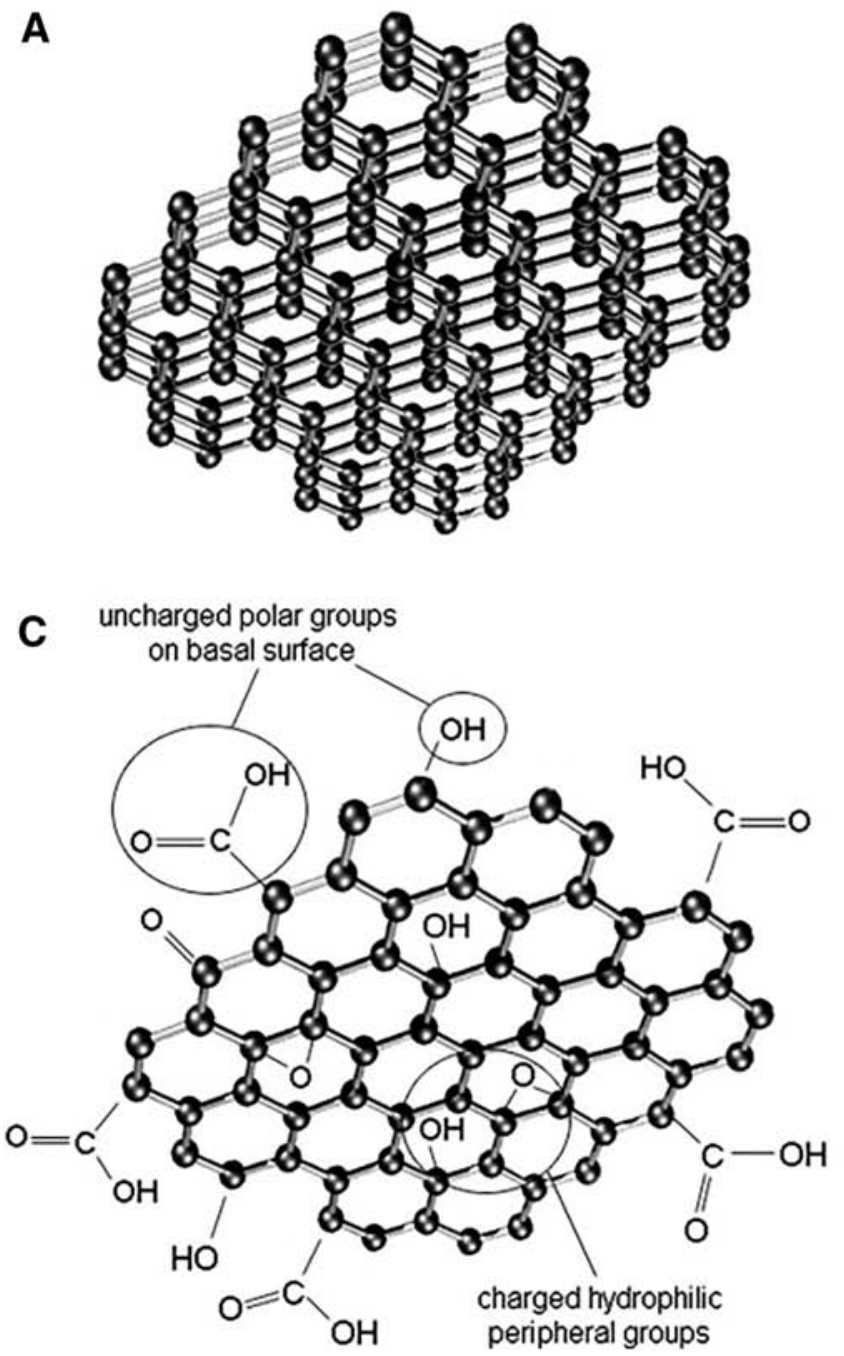

B

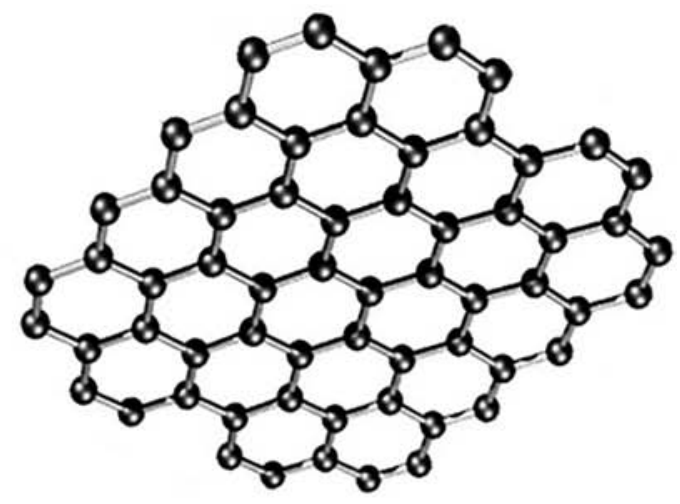

D

hydrophobic m-bond
capable graphene domains

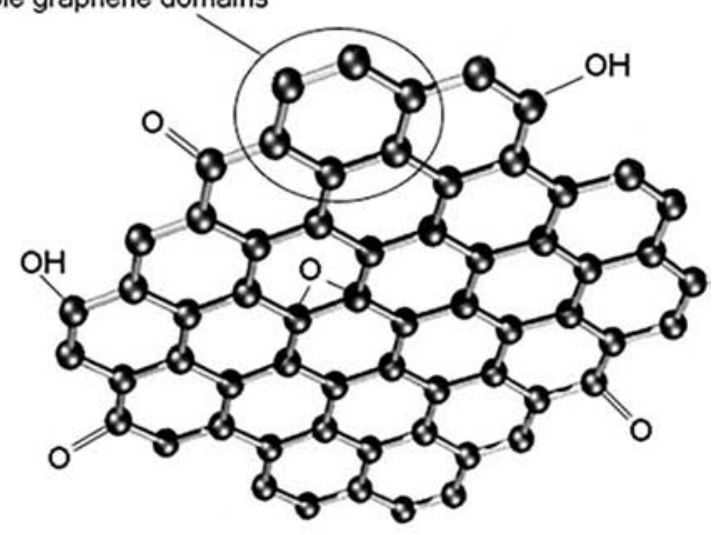

Figure 2. The graphene family: few-layered graphene (A), graphene nanosheet (B), GO (C), and rGO (D). Image reproduced with permission of Springer. ${ }^{34}$

oxygen-containing groups cause most of the carbon atoms to remain in $\mathrm{sp}^{2}$ hybridization in the GO layers. Because of these differences in hybridization patterns, orientation and defects, layer surfaces are rougher in the GO sheets. These GO sheets can be also partially reduced to the so-called rGO sheets by removing oxygen-containing functional groups.

Graphene has a relatively high Young's modulus, effective moisture barrier and fine electron mobility, which provides it with high electrical and thermal conductivity. ${ }^{37}$ As a rolled-up graphene sheets, CNTs have similar strength and stiffness with graphene but the conduction properties are different (i.e., metallic or semi-conducting). The thermal conductivity of $\mathrm{CNT}$ is comparable to graphene and the mobility of graphene is higher than $\mathrm{CNT}^{38}$

The GO can be produced with high yields by using inexpensive graphite. It is highly hydrophilic and provides $\mathrm{pH}$ dependent negative surface charge and colloidal stability. The GO has a higher surface area than other carbon-based nanomaterials and unique amphiphilic surface properties that allows the adsorption of proteins, dye molecules and water-insoluble drugs through noncovalent interactions. Thanks to its fine-tuning ability, GO can be used in smart materials and systems that allow control over the release of small molecules. ${ }^{39}$

\section{Production of graphene and graphene oxide}

Numerous methods have already been established for producing various kinds of materials from the graphene family (Figure 2). These methods can be divided into two major approaches, top-down and bottom-up (Figure 3). ${ }^{40}$ Top-down approaches include mechanical exfoliation, ${ }^{41}$ electrochemical exfoliation, ${ }^{42}$ solvent-based exfoliation ${ }^{43}$ and unzipping $\mathrm{CNTs}^{44}$; while epitaxial growth ${ }^{45}$ and $\mathrm{CVD}^{46}$ are among the bottom-up approaches. These different techniques not only yield graphenes with various sizes, shapes and compositions in various environments, but also provide different opportunities for the material's subsequent functionalization.

In the micromechanical cleavage method, graphene is isolated from graphite by using adhesive tape ${ }^{47}$; and mono-, di-, and few-layer graphene sheets are produced through repeated cleavage. Due to its slow production process, this technique provides high quality sheets and it is usually preferred if the fundamental properties of graphene are only investigated. 


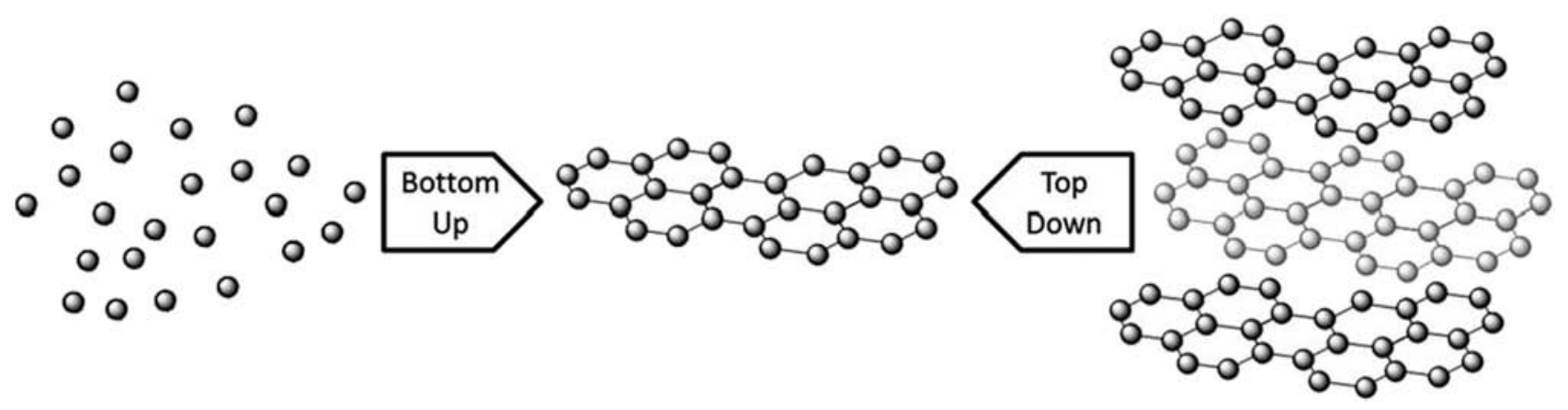

Figure 3. A schematic of bottom-up and top-down approaches for graphene synthesis. Image reproduced with permission of The Royal Society of Chemistry. ${ }^{40}$

Mechanical exfoliation is the initial step of the numerous discoveries of electronic and mechanical properties of graphene and the development of new production methods. ${ }^{48}$ Exfoliation of graphite involves the weakening of van der Waals forces to separate the layers from each other. In electrochemical exfoliation, diluted $\mathrm{H}_{2} \mathrm{SO}_{4}$ or $\mathrm{KOH}$ are used as a reactive sacrificial electrode solvent. Graphene is collected from this electrolyte solution that contains pyrene derivatives, $\mathrm{H}_{2} \mathrm{SO}_{4}$, $\mathrm{KOH}$, or surfactants such as sodium cholate, cetyltrimethylammonium bromide, as bilayer or few-layer sheets. ${ }^{49}$ With this approach, a mixture of different thicknesses of graphite sheets can be isolated by centrifugation. However, it is difficult to remove surfactant molecules, which might influence the electrical and electrochemical features of graphene. ${ }^{50}$

Solvent-assisted or thermal exfoliation of graphite ${ }^{43}$ is based on increasing the total area of graphite crystallites via immersing the graphene precursor into a solvent with sufficient surface tension. ${ }^{32}$ The Hummers method is commonly used for GO exfoliation. ${ }^{51}$ This procedure involves the oxidation of graphite in $\mathrm{H}_{2} \mathrm{SO}_{4}$ with $\mathrm{KMnO}_{4}$ as an oxidant for the reaction at $45^{\circ} \mathrm{C}$. Reduced GO is obtained following the exfoliation of GO. This method is highly popular and can yield graphene derivatives in addition to pristine graphene, but it also suffers from a high number of defects due to the harsh conditions of the production process. In addition, removing the expensive and hazardous components of the reaction is difficult, which may affect the final properties of graphene. Depending on the concentration of graphene, a decrease in flake size and increase in defect contamination might also occur.

Another way of producing graphene and GO is longitudinally cutting (or "unzipping") CNTs. ${ }^{44}$ The unzipping method allows for better control and chemical functionalization capacity compared to other fabrication techniques. The graphene nanoribbons that are produced using the unzipping method were found to be conductive; however, they are electronically inferior compared to the large-scale graphene sheets because of the presence of oxygen defect sites. ${ }^{52}$ Jiao et al. have showed that graphene nanoribbon formation can start from a dispersion of MWCNTs and proposed an unzipping opening mechanism that involves the exposure of a poly(methyl methacrylate) (PMMA)-MWCNTs film to an Ar plasma for varying time periods (Figure 4). ${ }^{44}$

The four aforementioned techniques constitute the major top-down synthesis methods of graphene and its derivatives. As a bottom-up fabrication technique, the epitaxial growth technique entails the sublimation of $\mathrm{Si}$ atoms and layer-by-layer reorganization of carbon atoms. ${ }^{46}$ In this method, thin layers of graphene form on the entire surface of silicon carbide ( $\mathrm{SiC}$ ) wafers at precisely defined time and temperature intervals. ${ }^{53}$ This provides relatively high quality, but graphene sheets with more than two layers can rarely be obtained as side products. Nevertheless, some studies of these structures have revealed the presence of numerous holes and cavities on the graphene surface due to very weak bonding and rotation of the individual layers, which cause high surface roughness. ${ }^{54}$ New methods have been developed to obtain more stable and homogeneous monolayers, which generally involve the formation of graphene on $\mathrm{SiC}$ at high temperatures $\left(>1000{ }^{\circ} \mathrm{C}\right)$ and under ultrahigh vacuum conditions. ${ }^{50} \mathrm{SiC}$ substrates designed for the production of graphene layers are commercially available; however, they are too expensive for commercial applications.

$\mathrm{CVD}^{55}$ is another useful bottom-up method for the production of graphene materials. Graphene that is produced by this technique has better crystallinity compared to those produced through other methods. ${ }^{35}$ Uniform polycrystalline graphene films with large surface areas are grown on $\mathrm{Cu}$ foils and films of metal such as $\mathrm{Ni}, \mathrm{Cu}, \mathrm{TiO}_{x}$, and so on, by CVD. ${ }^{46}$ Even though the complete process usually requires transfer from the copper support to a dielectric surface or other substrate of interest, square meters of graphene production has been achieved using this technique. ${ }^{56}$ CVD can also be used to fabricate monolayer graphene with high quality. The major drawback of this method is that the synthesis requires long period of time due to the difficulty in separation of the layers. A general comparison of advantages and disadvantages of the methods used to produce graphene are shown in Table 1.

\section{Functionalization strategies}

\section{Functionalization of carbon nanotubes}

Pristine CNTs have limited solubility and tend to aggregate in most types of solvents and biological media due to strong $\pi-\pi$ stacking and van der Waals interactions. Their limited water solubility makes them difficult to process and restricts their use for biomedical applications. For this reason, it is necessary to functionalize CNTs to improve their water dispersibility and biocompatibility. It should also be mentioned that the surface 


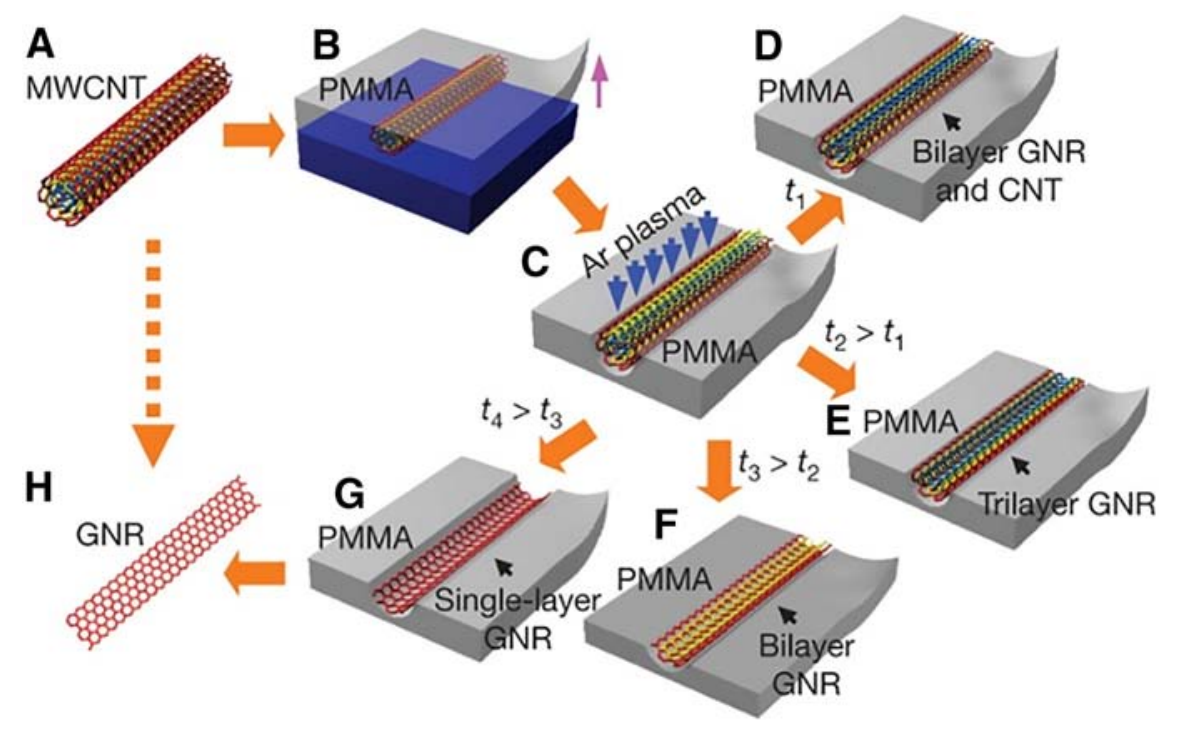

Figure 4. Graphene nanoribbon formation from MWCNTs that are embedded in PMMA and then treated with an Ar plasma. Reprinted by permission from Macmillan Publishers Ltd: [Nature], ${ }^{44}$ Copyright (2009).

chemistry of CNTs highly affects their behavior in vitro (e.g. for cellular uptake) and in vivo (e.g. for blood circulation time and biodistribution). ${ }^{57}$

CNTs exhibit chemical reactivity to many reactants depending on both their aromaticity and diameter and might be more reactive at their ends than in areas along the sidewalls due to increased curvature at the terminal caps. ${ }^{58}$ In addition, reactive locations mainly exist at or close to structural defects, including topological defects, points of rehybridization, vacancies in the CNT lattice and substitutional dopant impurities, which are formed during the fabrication process or occur during post-processing treatments such as purification and separation. ${ }^{59}$ These defects are a possible starting point for functionalization, and the high specific surface area of CNTs enables the adsorption or covalent bonding of various structural motifs at a high density.

In summary, considerable effort has been spent to develop novel functionalization methods. Main approaches for the functionalization of CNTs can be generally classified in three categories: covalent, noncovalent, and endohedral functionalization. ${ }^{60}$ The present section details these strategies, with emphasis on the methods that are especially suitable for biomedical applications.

\section{Covalent functionalization of carbon nanotubes}

The covalent modification of CNTs provides stronger bonds between the CNT and desired functional groups and offers a greater measure of control compared to methods based on noncovalent functionalizations. However, this approach changes the conjugated $\pi$-electron framework of CNTs (by hybridization from $\mathrm{sp}^{2}$ to $\mathrm{sp}^{3}$ ) and introduces defects onto the sidewalls of nanotubes, resulting in the loss of some intrinsic properties such as NIR fluorescence and Raman scattering characteristics. Therefore, covalent functionalization of CNTs has been widely utilized for drug and gene delivery, but is usually not ideal for sensing and bioimaging applications. ${ }^{21}$ Various strategies are used for the covalent functionalization of CNTs for biomedical purposes, including surface oxidation of CNTs, cycloaddition reactions, radical additions and their subsequent derivatizations with biologically relevant molecules. ${ }^{60}$

The most common method involves reactions with carboxylate groups generated on both the sidewall and nanotube ends of CNTs by oxidative treatment (Figure 5,A) under a wide variety of experimental conditions, such as sonication, refluxing in oxygen-containing acids (e.g., $\mathrm{HNO}_{3},{ }^{61} \mathrm{HNO}_{3} / \mathrm{H}_{2} \mathrm{SO}_{4},{ }^{62}$ and $\mathrm{H}_{2} \mathrm{SO}_{4} / \mathrm{KMnO}_{4}{ }^{63}$ ) and applying ozone or $\mathrm{H}_{2} \mathrm{O}_{2}{ }^{63}$ However, the oxidation treatment can shorten the length of CNTs and open up the end caps as a direct consequence of functionalization by oxygen-containing groups. For further derivatizations, these groups are used as anchor sites for esterification or amidation reactions (Figure $5, B$ ), which are widely used for the conjugation of water-soluble organic molecules, hydrophilic polymers like polyethylene glycol (PEG), nucleic acids (DNA or RNA), or peptides, resulting in the production of multifunctional CNTs. ${ }^{60}$

Another widely used method involves direct additional reactions such as 1,3-dipolar, nitrene and carbene cycloaddition reactions. The cycloaddition reactions occur uniformly and densely at the CNT sidewalls, rather than the ends or defect sites (as is the trend for the oxidation functionalization strategy), and provide remarkable solubility in water, many organic solvents and physiological conditions. ${ }^{64}$ 1,3-dipolar cycloaddition of azomethine ylides results in the formation of pyrolidine rings on the CNT surface (Figure 5, C), which can be substituted with many functional groups like amino acids, peptides, ${ }^{65}$ therapeutic agents ${ }^{66}$ and fluorescent molecules ${ }^{67}$ for diverse biomedical applications.

The nitrene addition on the sidewall of CNTs is achieved by reactive alkyloxycarbonyl nitrenes obtained from alkoxycarbonyl azides through thermal decomposition or photolysis of organic azides (Figure $6, D$ ). ${ }^{68} \mathrm{~A}$ broad range of nitrene precursors are used for the covalent binding of a variety of 
Table 1

Advantages and disadvantages of techniques currently used to produce graphene. ${ }^{40,50}$

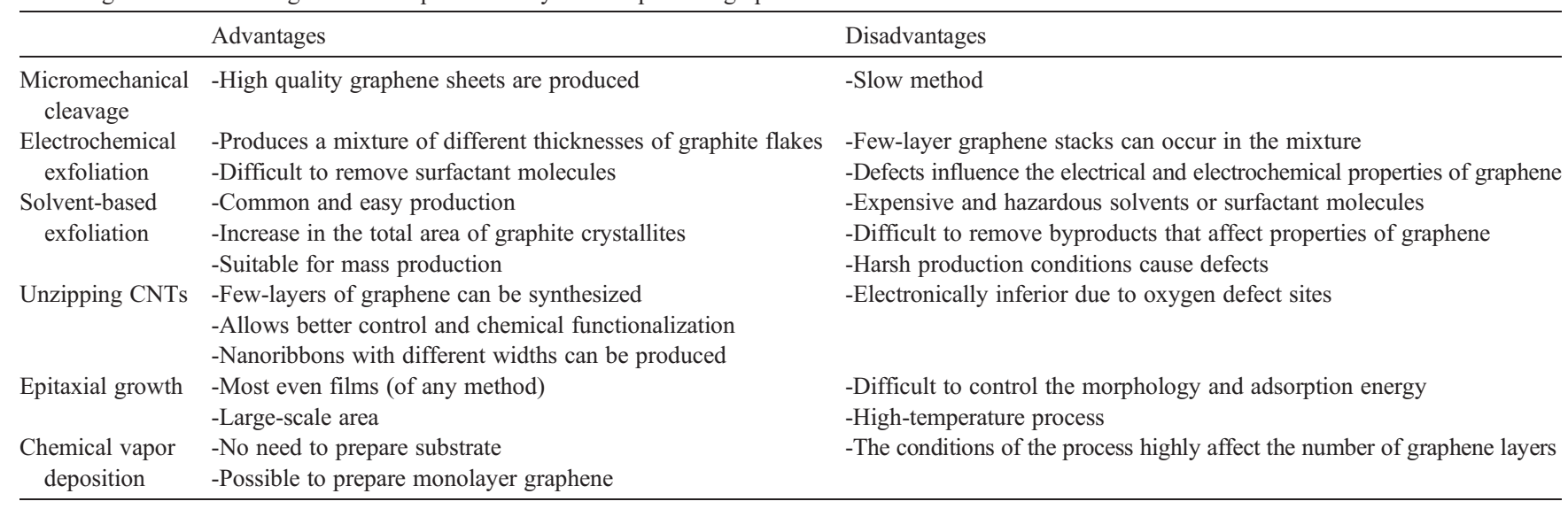

different groups. The dichlorocarbene moiety has also been conjugated onto the CNTs using a chloroform $/ \mathrm{NaOH}$ or a phenyl(bromodi-chloromethyl) mercury reagent (Figure 5, E), but X-ray photoelectron spectroscopy (XPS) results suggest that the degree of functionalization is low. ${ }^{69}$

Aryl diazonium coupling is frequently used to covalently functionalize CNT surfaces via radical addition reactions because of its simplicity and high yield. In this method, aryl diazonium salts are prepared ex situ from tetrafluoborate salts (Figure 5, F) or formed in situ via the reaction of aromatic amines with isoamyl nitrite and/or with $\mathrm{NaNO}_{2}$ in ionic liquids (or even without a solvent). ${ }^{18}$ The reaction rate depends on the metallic character of CNTs. On the other hand, this technique has been only rarely employed for biological applications. Further details about covalent functionalization can be obtained from the relevant reviews. ${ }^{70}$

\section{Noncovalent functionalization of carbon nanotubes}

Noncovalent functionalization of CNTs is a useful and convenient technique to obtain highly dispersible and processable CNTs without disturbing the carbon lattice, typically through the attachment of amphiphilic molecules ranging from small molecules to polymers. The principal advantage of this method is that it preserves the structure and optical properties of CNTs. Ideally, noncovalent functionalization should provide higher water solubility, biocompatibility, stability in various biological solutions and functional groups that are available for further bioconjugation. ${ }^{57}$ Hydrophobic interactions, $\pi$-stacking and/or van der Waals forces between CNTs and guest molecules are the driving forces for this method.

The high specific surface area of CNTs provides high loading capacity for adhering molecules. ${ }^{60}$ Many amphiphilic molecules, including pyrene, naphthalene derivatives, proteins, RNA, DNA, peptides, polymers and surfactants have been successfully adsorbed or wrapped onto the CNTs through noncovalent interactions. $^{70}$ The dispersion of the CNTs usually depends on the chemical characteristics of nanotube surfaces, the type and concentration of the amphiphilic molecule and the solvent, and dispersing conditions such as ultra-sonication and temperature. Steric stabilization by adsorbed nonionic surfactants or polymer layers is dominant if nonionic surfactants or polymers are used. In contrast, ionic amphiphilic molecules facilitate the dispersal of CNTs through electrostatic repulsion between similarly charged groups that are oriented towards (or away from) the solution. ${ }^{72}$

Paloniemi et al. reported that specific interactions, like charge transfer and ion- $\pi$ interactions, between CNTs and surface elements are important for the attachment of small aromatic molecules. The morphology of the aromatic moiety also affects the strength of $\pi-\pi$ interactions by altering the curvature of the nanotube surface. ${ }^{73}$ In particular, molecules containing polyaromatic components generally demonstrate a stronger affinity towards the basal plane of CNT surfaces compared to single aromatic moieties. This effect is readily observed in pyrene-containing molecules, which have attracted considerable attention as noncovalent modification agents in recent studies. ${ }^{74}$

Cationic, anionic or nonionic surfactants have been successfully used to produce stable aqueous dispersions of CNTs. The acquisition of stable CNT dispersions depends strongly on the length of the hydrophobic regions and the structure of the hydrophilic head group of the surfactant. However, surfactants face several problems in biological environments, including high critical micellar concentrations, lower stability and limited interaction with cellular proteins. Many of these problems can be circumvented by the use of PEG-modified phospholipids, which are non-toxic, biocompatible and amphiphilic polymers that possess various functional groups that can be further functionalized with therapeutic and targeting molecules. ${ }^{75}$

The potential of peptides as noncovalent modifiers for CNTs has been explored through the development of novel bioactive nanomaterials and may lead to new advances in biosensor and tissue engineering applications. ${ }^{76}$ Peptides containing histidine and tryptophan residues at specific locations were identified by phage display to exhibit specific affinity to CNTs. ${ }^{77}$ In addition, successful CNT dispersions have been prepared with the aid of amphiphilic peptide sequences that contain phenylalanine at specific locations ${ }^{78}$ and fold into $\alpha$-helixes on CNT surfaces. Self-assembling peptide amphiphile molecules, containing charged amino acid sequences covalently coupled to either a hydrophobic alkyl tail ${ }^{79}$ or phenylalanine, ${ }^{76}$ have also been used to disperse MWCNTs in aqueous solutions, with stable 


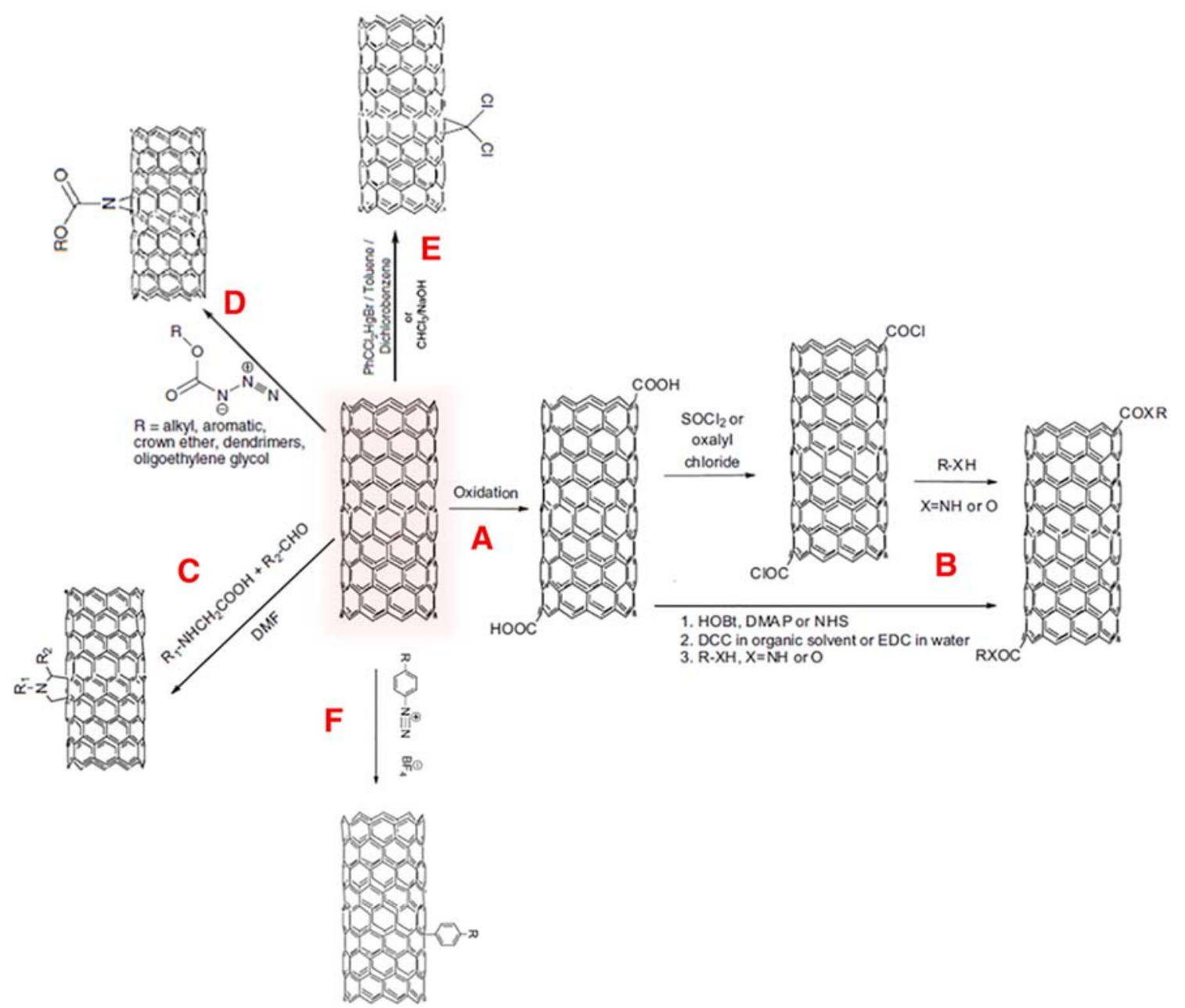

Figure 5. Covalent functionalization of CNTs; oxidation (A) and further esterification and amidation reactions (B), 1,3 dipolar cycloaddition (C), nitrene cycloaddition (D), carbine cycloaddition (E) and radical addition of aryl diazonium salt (F). Images reprinted and adapted with permission from The Royal Society of Chemistry. ${ }^{18,71}$

dispersions being obtained when the noncovalently functionalized CNTs were highly charged. As amphiphilic polypeptides, proteins have also been widely used to disperse CNTs, and their performance depends on diverse parameters including primary structures and $\mathrm{pH}$. But direct noncovalent modification of CNTs with proteins may partially unfold them and results in the loss of their biological functions. Nevertheless, indirect adsorption of proteins is possible with the aid of linker molecules such as 1-pyrenebutanoic acid succinimide ester, which immobilize proteins onto the CNT surface while maintaining the native protein structure. ${ }^{80}$

\section{Endohedral functionalization of carbon nanotubes}

In addition to the functionalization of the outer surface of CNTs, molecules can also be encapsulated within the hollow cavity formed by the nanotubes. Depending on the physicochemical properties and stability of the filler molecules, several endohedral encapsulation strategies are used for this purpose. Some of these strategies include high-temperature processes such as the CVD of filler metals, melting and capillary encapsulation of metals, gas phase filling-polymerization of polycyclic aromatic molecules, and in situ thermal decomposition of organometallic precursors. Metal and magnetic compound-filled CNTs obtained by these methods are useful for some biomedical (i.e., theranostics) applications; however, milder reaction conditions are required to insert biomolecules inside the cavities of CNTs. Supercritical $\mathrm{CO}_{2}$ extraction, nano-extraction and nano-condensation are among the methods used for the encapsulation of heat-sensitive biomolecules. Detailed information about this approach can be found in recent review. ${ }^{81}$

\section{Functionalization of graphene and graphene oxide}

Functionalization of graphene and GO can be either covalent or noncovalent. Covalent functionalization of GO can be performed through functional oxygen groups on the surface, carboxylic acid groups on the edges or epoxy/hydroxyl groups on the basal plane. Covalent functionalization can be achieved by several chemical reactions such as nucleophilic substitution, electrophilic substitution, condensation, and addition reactions. Nucleophilic substitution reactions target the epoxy groups of 

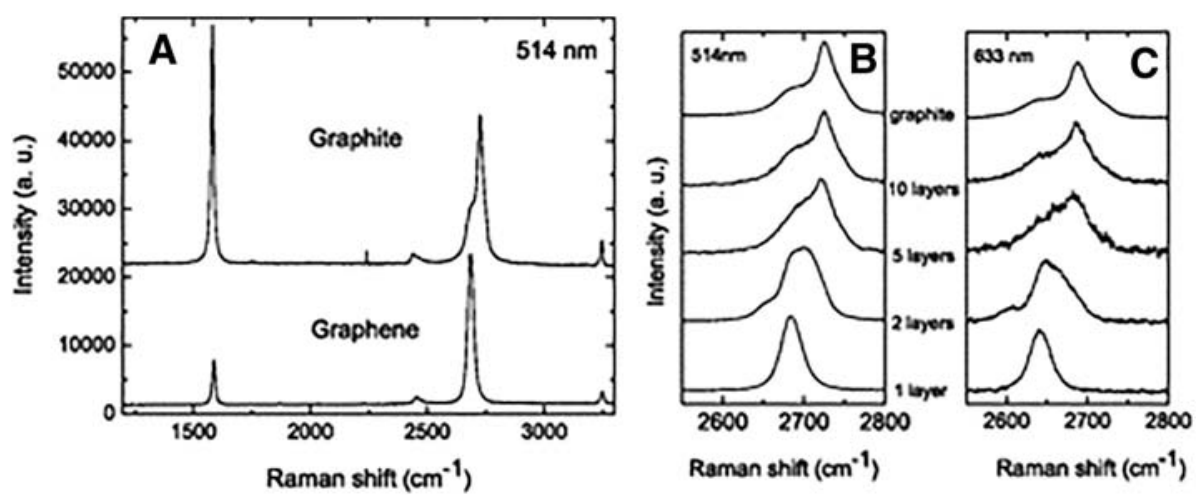

Figure 6. Raman spectra at $514 \mathrm{~nm}$ for bulk graphite and graphene (A), change of the spectra at $514 \mathrm{~nm}$ with the number of layers (B), change of the Raman spectra at $633 \mathrm{~nm}$ with the number of layers (C). Reprinted and adopted with permission from the American Physical Society, ${ }^{99}$ Copyright (2006).

GO. A variety of groups, such as alkyl amines, amino acids, ${ }^{82}$ dopamine $^{83}$ and polyglycerol, ${ }^{84}$ can be substituted in this manner. Regarding the hydrophilic nature of GO, it is able to conjugate amino acids through the epoxy groups of GO and obtain a flat orientation of the functional groups on the surface of the GO as a result of a nucleophilic reaction in alkaline solution. ${ }^{82}$ In another example, chemically converted graphene sheets were produced from the reaction between 3-aminopropyltriethoxysilane (APTS) and GO. ${ }^{85}$ These sheets can be homogeneously dispersed in common solvents.

Functionalization through noncovalent interactions is achieved by several approaches such as polymer wrapping, adsorption of small molecules, and interaction with host molecules like porphyrins or biomolecules like deoxyribonucleic acid (DNA) and peptides. ${ }^{33}$ The functionalization of $\mathrm{GO}$ with these molecules is relatively common and well-characterized in the literature, though noncovalent modifications with various agents such as poly(sodium 4-styrenesulfonate), ${ }^{86}$ pyrenebutyric acid ${ }^{87}$ and amine terminated polymers ${ }^{88}$ have also been demonstrated. In addition, Liu et al. have reported a noncovalent functionalization technique for graphene, based on $\pi-\pi$ interactions between the $\pi$ orbitals of graphene and poly(N-isopropylacrylamide). ${ }^{89}$ Yang et al. have reported the supramolecular functionalization of $\mathrm{rGO}$ by the conjugation of poly(2,5-bis(3-sulfonatopropoxy)-1,4-ethynylphenylene-alt-1, 4-ethynylphenylene) polyelectrolyte through electrostatic interactions ${ }^{90}$ which, imparted long dispersion stability and high electrical conductivity to the material.

\section{Characterization}

The characteristic properties of carbon based nanostructures require characterization after synthesis or functionalization processes to determine their structural, morphological, thermal and electrical properties. For biomedical applications, conventional in vitro studies are usually performed in 2D plate cultures as opposed to the deposit forms used in in vivo studies. Classical techniques used for characterization of biomaterials are performed in 2D forms and are a representative of 3D assembly formed by these nanomaterials.
Scanning electron microscopy (SEM) and transmission electron microscopy (TEM) are widely used for the preliminary evaluation and determination of the morphology of CNTs and their impurities. TEM provides more accurate information on the diameter, number of walls, structural integrity and defects of the nanotube architecture. Energy-dispersive spectroscopy can also be used in conjunction with SEM and TEM to determine not only the metallic impurity content but also non-metallic and heteroatom identity and quantity after functionalization of carbon-based nanomaterials. After functionalization, if the attached groups are large enough to be resolved and/or have enough contrast against the CNT background, they can also be identified morphologically by SEM and TEM. ${ }^{27}$ Grazing incidence small-angle X-ray scattering allows the real time in situ study of growing nanotubes on substrates and provides information about the three-dimensional structure and orientation of CNTs, as well as morphological and structural data. It is also a very useful method if the nanostructures prove difficult to visualize by conventional means. ${ }^{91}$

Microscopic corrugations of graphene and its derivatives with a lateral dimension of 8 to $10 \mathrm{~nm}$ and height displacements of 0.7 to $1 \mathrm{~nm}$ can be estimated by TEM. ${ }^{92}$ Although some scanning tunneling microscopy imaging studies indicated a limited correlation between $<0.5 \mathrm{~nm}$ height corrugations and local electrical properties, ${ }^{93}$ bigger ripples $(2-3 \mathrm{~nm}$ in height) appear to be indicative of strain-induced local conductance modulations. ${ }^{94}$ Computational models, such as molecular dynamics simulations, can be employed to understand the mechanical properties of graphene monolayers. ${ }^{95}$ Experimentally, the Young's modulus of a few layers of graphene was also investigated by force-volume measurements in AFM. In a recent study, for defect-free graphene, a Young's modulus of 1.0 TPa and a fracture strength of $130 \mathrm{GPa}$ were determined by nanoindentation using AFM. ${ }^{96}$ In another study, the average elastic modulus and the highest fracture strength of GO platelets were reported as $\sim 32 \mathrm{GPa}$ and $\sim 120 \mathrm{MPa}$, respectively. ${ }^{97}$

Fourier transform infrared (FTIR) spectroscopy allows the qualitative identification of functional groups on carbon nanostructures through the interpretation of characteristic adsorption bands. However, FTIR spectra often have insufficient 
quality due to the strong electronic adsorption and light scattering properties of carbon nanostructures. To overcome this undesired behavior, the sample should have the smallest possible particle size. Attenuated total reflectance and diffuse reflectance methods can also be used to obtain higher quality spectra, and nuclear magnetic resonance spectroscopy can be used to confirm the presence of specific functional groups on nanostructures. XPS provides further insight into the chemical composition and environment of nanoparticle surfaces, but is limited to a depth of less than $10 \mathrm{~nm}$ into the structure.

Raman spectroscopy is a powerful tool to distinguish between the two distinct $\mathrm{sp}^{2}$ carbon nanostructures that characterize CNTs and graphene. Useful information about crystallite size, clustering of the $\mathrm{sp}^{2}$ phase, the presence of $\mathrm{sp}^{2}-\mathrm{sp}^{3}$ hybridization and chemical impurities, the magnitude of the mass density, the optical energy gap, elastic constants, doping, defects and other crystal disorders, edge structure, strain, number of graphene layers, nanotube diameter, chirality, curvature, and metallic/semiconducting behavior can be obtained utilizing Raman spectroscopy. ${ }^{98}$ The Raman spectrum of carbon materials typically yields three main characteristic bands; namely D, D' and G modes in the 1000 to $2000 \mathrm{~cm}^{-1}$ range and the overtone and combination bands $\mathrm{G}^{\prime}=2 \mathrm{D}$ in the 2400 to $3400 \mathrm{~cm}^{-1}$ range. The $\mathrm{G}$ mode is centered around $1590 \mathrm{~cm}^{-1}$ and attributed to the graphitic structure. D and D' modes arise from amorphous disorder and defects in the carbon lattice. The crystallite and amorphous region densities of the structure are calculated from the intensity ratio of $\mathrm{D}$ and $\mathrm{G}$ modes $\left(\mathrm{I}_{\mathrm{D}} / \mathrm{I}_{\mathrm{G}}\right)$. The radial breathing mode (RBM) includes various vibrational transitions of radial expansion and contraction of the CNTs, and is usually observed in the region between 100 and $350 \mathrm{~cm}^{-1}$. The frequency of RBM is inversely proportional with the diameter of nanotubes. ${ }^{27}$ With this tool, the quality of the graphene sheets can also be determined by evaluating the extent of disorder and number of defects on the graphene surface. In addition, the number of the layers can be determined by the shape, width, and position of the 2D peak. ${ }^{99}$ Graphene and its derivatives exhibit prominent graphite peaks that are known as the "G band" (at $1580 \mathrm{~cm}^{-1}$ ) and the " $2 \mathrm{D}$ peak" (at around $2700 \mathrm{~cm}^{-1}$ ). ${ }^{92}$ Ferrari et al. demonstrated that increasing the number of graphene layers causes the 2D peak to shift to higher wavelength ranges (Figure 6). ${ }^{99}$

Thermogravimetric analysis (TGA) measures the mass change of a sample, which is examined as a function of temperature under a pre-defined atmosphere. It is also possible to identify the gas phase composition of the sample with the aid of TGA in conjunction with FTIR. TGA is frequently utilized to evaluate the thermal stability of carbon nanostructures, and also provide information about the proportion of carbon and other impurities in the CNTs. Additionally, TGA can measure the degree of functionalization due to the loss of organic moieties that occurs when functionalized carbon nanostructures are heated in an inert atmosphere. ${ }^{100}$

\section{Biocompatibility}

\section{Cytotoxicity}

Carbon-based nanomaterials have been widely researched in biological systems in recent years due to their diverse potential and unique physicochemical and morphological structures.
Unlike graphite, which is a naturally occurring stable form of carbon; graphene, GOs and CNTs are artificially synthesized materials with uncertain effects on biological systems. Consequently, the toxicity of carbon-based nanomaterials has become a topic of great interest, and considerable effort has been spent to characterize their cytotoxic effects and behavior in microorganisms, cells and organisms, both to evaluate the risks associated with their spread to the environment and to determine their potential utility in biotechnological and biomedical applications.

In general, there are several factors that affect the toxicity of nanoscale biomaterials such as graphene, GO and CNTs; including size, number of layers, shape, structure, purity, exposure route, dose, charge, structural defects and hydrophobicity. ${ }^{101}$ Surface hydrophobicity of a material directly affects its dispersion characteristics under physiological conditions. As such, surface functionalization of carbon nanomaterials can greatly alter their bioaccumulation, toxicity and cellular and systemic responses. Both pristine graphene and CNTs are highly hydrophobic, and functional groups are often used to increase the water solubility of these materials, which also enhances their absorption in biological context.

The main cellular uptake mechanisms for graphene are receptor mediated endocytosis, phagocytosis and membrane adsorption, whereas CNTs are internalized via micropinocytosis, receptor-free endocytosis and piercing (rupturing and self-healing of cellular membrane) in addition to the same internalization pathways as graphene. ${ }^{102}$ The GO is also known to be internalized via non-phagocytotic pathways as well as phagocytotic pathways. ${ }^{103}$ Pristine graphene has been shown to display lower dispersibility, inducing oxidative stress at $10 \mu \mathrm{g} / \mathrm{mL}$ and leading to apoptosis in neural-like cells (PC-12) in a dose and time dependent manner. Graphene exposure was also associated with an increase in the level of reactive oxygen species (ROS) production and cell death through the induction of MAP kinases and the transforming growth factor beta pathway in macrophages. Graphene was initially observed to cause stronger metabolic activity compared to SWCNTs at lower concentrations; however, this trend was reversed at higher doses. ${ }^{104}$ In another study, pristine graphene was reported to cause toxicity by accumulating on the cell membrane and eliciting an apoptotic response due to oxidative stress, whereas carboxyl-functionalized hydrophilic graphene was internalized normally and exhibited no cytotoxicity. ${ }^{105}$ Both GO and carboxylated graphene was observed to be toxic to nonphagocytotic epithelial lung carcinoma cells by penetrating through the membrane, concentrating as vesicles in the cytosol, and increasing ROS; though lower concentrations of both materials $(<4 \mu \mathrm{g} / \mathrm{mL})$ did not exert any cytotoxic effect. ${ }^{106}$ However, incubation of graphene with $10 \%$ fetal bovine serum effectively decreased its toxicity, possibly because of the coating of the graphene surface with a protein layer. ${ }^{107}$ The GO was also reported to induce necrotic death in macrophages by causing the activation of Toll-like receptor-4 signaling and production of tumor necrosis factor alpha via autocrine signaling in vitro. ${ }^{108}$ The in vivo biocompatibility of the GO is not well understood. It was shown to be effectively and rapidly cleared from zebrafish without lasting effects, ${ }^{109}$ whereas lung administration of the GO to mice caused severe and persistent lung damage while pluronic dispersion of graphene minimized the damage. ${ }^{110}$ PEGylation is a common 
Table 2

The effects of $1 \mathrm{D}$ and 2D carbon nanomaterials and their functionalization types and dosage.

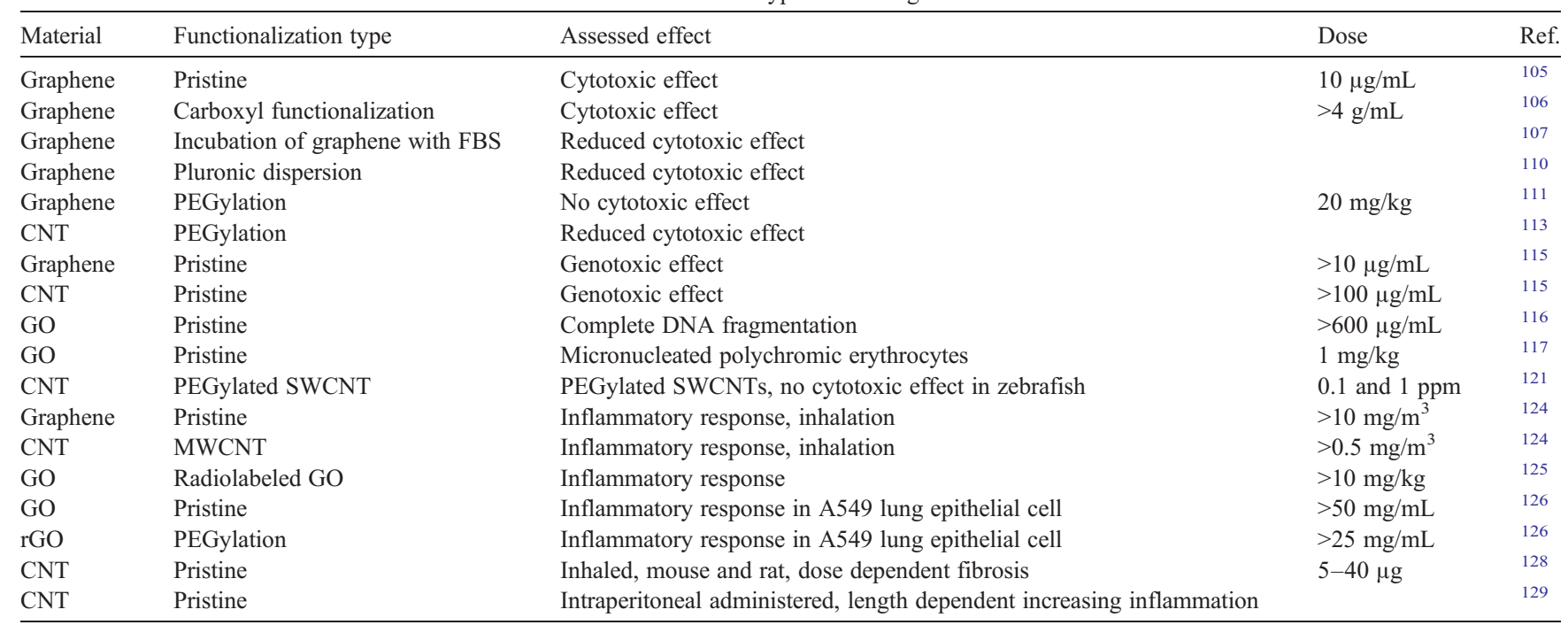

approach in order to mask the material against immune system and reduces the oxidation of graphene. No toxicity was observed in mice treated with PEGylated graphene $(20 \mathrm{mg} / \mathrm{kg})$, as observed in histological and hematological analyses. ${ }^{111}$

Pristine CNTs were also shown to cause cytotoxicity through inflammation, activation of the immune system, and production of ROS. However, surface functionalization can be used to eliminate some of these problems. Functionalization is usually performed to increase the hydrophilicity and water dispersion of CNTs, and plays a vital role in facilitating non-destructive interactions between nanomaterials and cellular interfaces. ${ }^{112}$ PEGylation of SWCNTs, for example, was reported to reduce their toxicity and downregulate ROS production-related pathways compared to uncoated SWCNTs, ${ }^{113}$ leading to lower cellular ROS levels. A number of studies hypothesize that surface functionalization improves the biocompatibility of 1D and $2 \mathrm{D}$ carbon nanomaterials ${ }^{111,114}$; however, some studies also suggest that the alteration of surface properties promotes and facilitates cellular uptake of the materials and increases their toxic potential. The assessed effect of 1D and 2D carbon nanomaterials having different functionalization type and dosage are summarized in Table 2. More comprehensive studies are necessary in order to understand the toxicity mechanisms of 1D and 2D carbon-nanostructures and to rationally design surface functionalization types that are able to mitigate the risks associated with these materials.

\section{Genotoxicity}

Genotoxicity is defined as the property of the material to directly or indirectly cause damage to the DNA. ROS produced in response to graphene-related materials can conceivably create an increase in cellular acidity, leading to DNA damage and possibly cancer. There are relatively few studies regarding the genotoxicity of graphene-based materials compared to cytotoxicity studies. Previously, the genotoxic potential of various nanoparticles and nanomaterials, including graphene, GO and
CNT were shown; and graphene exhibited genotoxicity at the lowest concentrations $(>1 \mu \mathrm{g} / \mathrm{mL})$. The CNTs, in contrast, produced adverse effects on the cellular genome at $>100 \mu \mathrm{g} / \mathrm{mL}$ concentration. ${ }^{115}$ Wang et al. showed that GO interacted with DNA in a concentration-dependent manner, completely fragmenting DNA at $600 \mu \mathrm{g} / \mathrm{mL}$. ${ }^{116} \mathrm{GO}$ also inhibits the $\mathrm{S}$ phase of the cell cycle and leads to cell cycle arrest at the $G_{0} / G_{1}$ phase. Intravenously injected $\mathrm{GO}$ at a dose of $4 \mathrm{mg} / \mathrm{kg}$ for 5 days induced micronucleated polychromic erythrocytes in mice. ${ }^{117}$ Akhavan et al. reported that rGOs elicited size- and concentration-dependent increases in DNA fragmentation and produced chromosomal aberrations in human mesenchymal stem cells (MSCs). ${ }^{118}$ Pristine CNTs have been shown to cause genotoxicity mainly due to their capability to cause increased ROS production, which further causes DNA breaks through oxidation. ${ }^{119}$ Poulsen et al. suggested that CNTs with smaller surface areas (and therefore larger sizes) are associated with increased genotoxicity, as observed in mice studies. ${ }^{120}$ However, a recent study by Girardi et al. suggests that embryonically applied PEGylated SWCNTs did not cause any genotoxicity in zebrafish between the concentration values of 0.1 and $1 \mathrm{ppm} .^{121}$

\section{Inflammatory response}

It is not completely clear what happens to the $1 \mathrm{D}$ and $2 \mathrm{D}$ carbon nanomaterials once they are administered into the body; however, the immune reactions that occur in the body strongly depend on the route of administration. Graphene sheets with PEG and radioactive iodine has been administered intravenously to mice and graphene sheets were found to accumulate in reticuloendothelial system, liver and spleen, followed by a gradual clearance after 315 days. ${ }^{122}$ Graphene and GO are more damaging when they are directly administered to the bloodstream or inhaled. Macrophages, as the first step of the immune defense machinery, respond immediately to foreign materials that are administered to blood. In addition, other immune cells such as phagocytes and dendritic cells are more sensitive to 
Table 3

Applications of 1D and 2D carbon nanomaterials and their utilized properties.

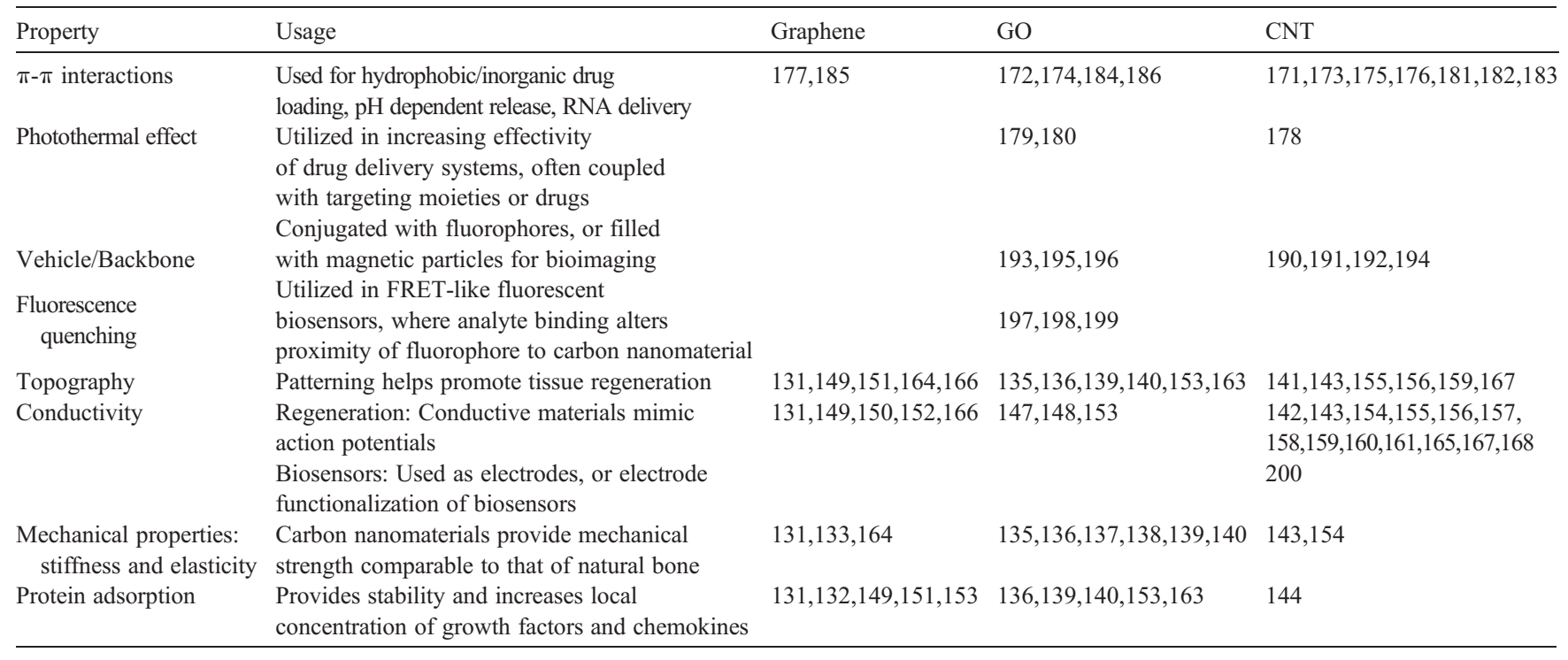

graphene than stem cells, osteocytes and chondrocytes. Wang et al. showed that the Th2 immune response was activated in the lungs of mice following the intravenous administration of graphene nanosheets, which was reflected by a strong neutrophilic influx and the secretion of interleukins IL-5, IL-13 and IL-33 in the bronchoalveolar lavage fluid. ${ }^{123}$ In a comparative study, mice were exposed to graphene-related materials by inhalation, and the inflammation process was initiated at concentrations of $10 \mathrm{mg} / \mathrm{m}^{3}$ for graphene and $0.5 \mathrm{mg} / \mathrm{m}^{3}$ for MWCNTs. ${ }^{124}$ On the other hand, the GO was observed to cause no immune response or systemically pathological changes following IV injection in mice below the concentrations of $10 \mathrm{mg} / \mathrm{kg}$, however, significant inflammatory and immune response was triggered by higher concentrations. ${ }^{125}$ In another recent study, non-functionalized rGOs were found to adhere to cell membranes, potentially binding Toll-like receptors and activating inflammatory responses mediated by $\mathrm{NF}-\kappa \mathrm{B}$; while PEGylated rGOs were directly internalized by the cells but nonetheless created a NF- $\kappa \mathrm{B}$ mediated inflammatory response. ${ }^{126}$

The SWCNT and MWCNT both caused immune activation, immune cell proliferation, secretion of inflammatory cytokines, chemokines, tumor necrosis factor- $\alpha$, IL1- $\beta$, monocyte chemotactic protein-1 and transforming growth factor-1 in RAW264.7 macrophages. ${ }^{127}$ Furthermore, the CNTs were observed to cause lung inflammation and fibrosis in mice and rats, as they accumulated in inflammatory cells and triggered cytokine and growth factor secretions in the lungs, resulting in an increase in bronchoalveolar lavage fluid and the formation of epithelioid granulomas in the lung parenchyma. ${ }^{128}$ In addition, intraperitoneal and intrapleural injections of CNTs were observed to cause inflammation and fibrosis in the regions in which they were administered. $^{129}$ Although it is clear that inflammation is a frequent result of CNT exposure, further molecular, cellular and animal studies are nevertheless necessary to better determine the pathways in which $1 \mathrm{D}$ and $2 \mathrm{D}$ carbon materials are able to elicit immune responses.

\section{Bioactivity and applications}

As discussed above, 1D and 2D carbon nanomaterials have a number of unique properties that enable them to be used in biomedical applications. We provide a brief summary of the mentioned properties, and how they are exploited for biomedical applications in Table 3 .

\section{Tissue engineering}

\section{Bone regeneration}

Major bone defects are one of the main challenges in bone tissue engineering and require implantation of either autologous bone grafts or scaffolds produced from various natural or artificial materials. To date, a variety of synthetic or natural materials have been used for promoting osteogenesis, however, further applicability and regenerative success of these materials depend on whether they meet some criteria. An ideal bone implant material should facilitate the attachment, proliferation and differentiation of the bone cells, as well as stimulating and sustaining a bioactive signal that promotes bone regeneration. Stem cell based-therapies are still being investigated for this purpose; however, these cells require in situ scaffolds that promote viability, attachment, spreading and differentiation in order to effectively replace lost tissue.

The 1D and 2D carbon nanomaterials serve as promising materials as bone scaffolds, coating materials and drug delivery agents to support bone regeneration due to their strong mechanical properties, excellent flexibility and nanotopography. Both graphene and CNTs exhibit high conductivity profiles, which have positive effects on the healing process. It has been suggested that osteoblast activity, adhesion and proliferation are modified by external electrical stimulation. ${ }^{130}$ Graphene, with its great conductivity, charge carrier mobility and tensile strength, is considered to have a great potential as a material for bone regeneration. ${ }^{96}$ The CVD graphene has been shown to increase the proliferation of mesenchymal stem cells and osteoblast-like 
SAOS-2 cells. ${ }^{131}$ Pristine graphene itself did not improve proliferation, however, the osteoblastic differentiation of stem cells was enhanced by the material. ${ }^{132}$ The $2 \mathrm{D}$ graphene layers also enhanced osteogenic commitment compared to GO and PDMS, ${ }^{133}$ and MSCs cultured on CVD graphene-coated glass and $\mathrm{Si} / \mathrm{SiO}_{2}$ surfaces were shown to express higher amounts of osteocalcin, a late osteogenic marker. ${ }^{133}$

The MSCs cultured on GO nanoribbons also exhibited a 3.7 fold increase in mineralization compared to the those cultured on PDMS and glass. ${ }^{134}$ These effects could be attributed to the high modulus stiffness and elasticity of graphene, as well as the lateral cytoskeletal tension that causes the induction of cytoskeletal organization in a way that promotes osteogenic differentiation. ${ }^{133}$ Combination of other molecules with GO has also been one of the common approaches. Chitosan-GO scaffolds were observed to significantly enhance osteoblast attachment, proliferation and extracellular matrix formation, ${ }^{135}$ and another, similar study reported higher cellular attachment, proliferation, growth and mineralization rates following the attachment of GO carboxyls to the amine groups of chitosan. ${ }^{136}$ Calcium phosphate-mineralized $\mathrm{GO} /$ chitosan scaffolds could also adsorb bone morphogenic protein-2 (BMP-2) encapsulated bovine serum protein nanoparticles and silver nanoparticles for bioactivity and anti-bacterial purposes, respectively. In vitro and in vivo studies showed that bone marrow stromal cell differentiation and proliferation were enhanced in the presence of the former system, contributing to improved bone regeneration. ${ }^{137}$ GO was also incorporated into a gelatin hydroxyapatite matrix in order to increase its mechanical strength, and the composite material was found to be biocompatible, biodegradable and capable of enhancing the osteogenic differentiation of human adipose derived MSCs. ${ }^{138}$ Osteoinduction of MSCs was also observed on another polymer, poly(lactic-co-glycolic) acid (PLGA), following enrichment with GO. ${ }^{139}$ The mechanism for the enhancement of osteogenic differentiation and related pathways is hypothesized to lie in the combination of physiochemical properties of $\mathrm{GO}$, including its stiffness, nanoscale roughness, presence of functional groups such as reactive oxygen, water retention capability and hydrophilic nature, as well as the direct adsorption of biomolecules onto the GO surface. ${ }^{140}$

The CNTs have also been used in the field of osteogenic regeneration, as their incorporation adds great strength to composite materials. Studies have shown that alkaline phosphatase activity, calcium deposition, and osteoblast cell adhesion were enhanced on CNT/polycarbonate urethane composite scaffolds. ${ }^{141}$ The conductive properties of CNTs are also attractive for the field of bone regeneration. Poly(lactic acid) (PLA)/MWCNT composite scaffolds were reported to show increased cell proliferation and extracellular matrix calcium deposition of osteoblasts, ${ }^{142}$ while electrospun PLA/ MWCNT composites induced cell alignment and proliferation in the presence of electrical stimulation. ${ }^{143}$ In addition, CNTs were found to be effective in stimulating osteocyte proliferation in a time dependent manner, despite some initial cell death. Macrophages and neutrophils have been suggested to remove the toxic potential of CNT substrates, allowing the subsequent proliferation of the osteoblasts. $^{144}$

\section{Neural regeneration}

The central nervous system has a very low regenerative capacity due to the inhibitory environment, low abundance of neural stem cells (NSCs) and their low potential to compensate the loss of neurons. Current clinical treatments for nerve injuries have not advanced beyond minimizing secondary damage following initial neuronal loss. The NSC transplants are used to compensate for the lost neural tissue; however, NSCs require constant stimulation in order to survive and selectively differentiate into astrocytes when transplanted, highlighting the need for developing biomaterials that promote NSC survival and neuronal differentiation. Therefore, it is essential to investigate the mechanisms of NSC differentiation and develop biomaterials for this purpose in order to effectively facilitate nerve regeneration. Although regeneration is more favorable in the peripheral nervous system, nerve gaps longer than $2 \mathrm{~cm}$ still require the artificial connection of distal and proximal nerve stumps in humans. As such, effective neural treatment strategies require the elimination of the inhibitory signals that prevent regeneration, and scaffold biomaterials that restore the interconnections between nerve stumps. ${ }^{145}$

The 1D and 2D carbon nanomaterials have attracted tremendous interest in nerve regeneration due to their high conductivity, nanotopography and their ability to be functionalized for specific purposes. For instance, carbon nanotubes provide topographical cues for neurons, resembling the extracellular matrix fibers in the native environment of the nervous system. While certain polymers are similarly conductive, the rigid structure of polymers is not optimal for culturing neurons, since neural substrates must be designed to have lower Young's moduli compared to other tissues. ${ }^{146}$ The effect of graphene-based materials on neural differentiation has been widely studied. Lie et al. showed that culturing NSCs on 3D porous graphene foams promoted their differentiation towards neurons rather than astrocytes. In another in vitro study, human NSCs grown on rGO sheets exhibited accelerated neural differentiation following pulsed laser stimulation. ${ }^{147}$ More specifically, Yang et al. proved that embryonic stem cells showed enhanced dopaminergic neural differentiation on GO sheets in a dose dependent manner, whereas graphene and CNTs did not have such an effect. ${ }^{148}$ In another study, graphene-poly( $\varepsilon$-caprolactone) (PCL) nanofiber hybrid systems were developed for guiding stem cell differentiation into oligodendrocytes in order to promote myelination. Without introducing differentiation inducers, NSCs were guided to differentiate into mature oligodendrocytes due to the presence of permissive surfaces for the adhesion of cells and proteins, as well as the conductivity of the scaffold system, which could potentially be used for the treatment of myelination disorders. ${ }^{149}$ Polypyrrole-functionalized graphene nanofibers were also developed for improving the optic nerve regeneration, and retinal ganglion cells showed enhanced viability and neurite outgrowth in the presence of electrical stimulation on these substrates. ${ }^{150}$ In an in vivo study, Zhou et al. showed that the implantation of colloidal graphene-coated electrospun microfiber networks into the subventricular zone prevented glial scars, decreased microglia and astrocyte activation, and supported neuroblast migration from the subventricular zone. ${ }^{151}$ A recent work further demonstrated that of the neurogenic differentiation of MSCs was promoted by electrical stimulation on rGO- poly(3,4-ethylenedioxythiophene) (PEDOT) hybrid microfibers. ${ }^{152}$ GO was also used for coating aligned PLLA nanofibrous scaffolds, and PC-12 proliferation and differentiation were found to be significantly improved on these scaffolds. ${ }^{153}$ 
Because of their conductive, fibrous and hollow structure, as well as their high surface to volume ratios, CNTs have been an attractive materials for neural regeneration studies. Materials such as polymers and biomolecules have been used to modify carbon nanotubes to effectively guide the outgrowth of neurons. Silk-CNT composites, for example, were able to enhance the neural differentiation and axonal lengths of human embryonic stem cells ${ }^{154}$; while $\mathrm{Si}_{2} \mathrm{O}$ cell culture surfaces patterned with double-walled CNTs and CNT layers were observed to promote neural differentiation and adhesion of Neuro-2a cells to a greater extent than individual groups of $\mathrm{CNT}$ and $\mathrm{Si}_{2} \mathrm{O}$ surfaces. ${ }^{155} \mathrm{In}$ another study, immature spinal cord neurons isolated from neonatal rat spinal cords showed rapid growth on pyrolidine-functionalized MWCNT coated surfaces. ${ }^{156}$ In an in vivo study, electrospun collagen-PCL-MWCNT fibrous scaffolds were shown to assist in the recovery of rat sciatic nerve defect models and prevented muscle atrophy. In the same study, the scaffold also supported Schwann cell adhesion and elongation. ${ }^{157}$ Freeze-dried silk/SWCNT/fibronectin-based nerve guidance conduits also showed higher nerve conduction velocities and more myelinated axons in a rat sciatic nerve deficient model. ${ }^{158}$ Furthermore, collagen isolated from rat tails was incorporated into composite hydrogels with CNTs and the composite system provided significant stimulation of MSC differentiation into the neural fate. ${ }^{159}$ Koppes et al. showed that SWCNTs incorporated into Matrigel ${ }^{\mathrm{TM}}$ hydrogels showed enhanced neurite outgrowth of dorsal root ganglia compared to SWCNT-free Matrigel ${ }^{\mathrm{TM}}$ group, while external electrical stimulation promoted outgrowth within SWCNT-free control groups. ${ }^{160}$ In a recent study by Usmani et al., $3 \mathrm{D}$ conductive MWCNT meshes were used to culture organotypic spinal cord slices that were allowed to spontaneously grow. 3D artificial scaffolds substantially boosted regrowth of nerve bundles into a random net (Figure 7). ${ }^{161}$

\section{Muscle regeneration}

Skeletal tissue is composed of bundles of muscle fibers, which in turn are clusters of fused myoblasts. Significant skeletal muscle loss may occur due to damage, trauma or surgery, and deficiencies in its regeneration may lead to permanent functional disabilities. ${ }^{162}$ Although minor muscle injuries are often repaired rapidly with no medical intervention, the regeneration of bulk muscle tissue still remains a challenge in tissue engineering. It is known that muscle tissue is electrically responsive, and electrical cues have been utilized to promote myotube formation and muscle contraction. The 1D and 2D nanomaterials, with their outstanding flexibility, high conductivity, great mechanical properties and ultralow density, bear great potential as substrates for muscle regeneration.

The GO was reported to accelerate the myogenic differentiation of $\mathrm{C} 2 \mathrm{C} 12$ cells due to better adsorption of serum proteins and nano-topographical cues. ${ }^{163}$ Electrospun PLGA-collagen impregnated GO fibers were also observed to promote adhesion and proliferation of $\mathrm{C} 2 \mathrm{C} 12$ cells. ${ }^{164}$ In another recent study, conductive PCL-graphene composite scaffolds promoted the adhesion and proliferation of $\mathrm{C} 2 \mathrm{C} 12$ cells, as well as inducing the differentiation of these cells into multinucleated myotubes in a concentration-dependent manner. ${ }^{165}$ Stem cell based-treatments remain as promising strategies for cardiac regeneration, but despite the ability of MSCs to differentiate into cardiomyocytes in vitro, their clinical efficacy is quite low. Park et al. demonstrated that MSC proliferation and commitment towards the cardiomyogenic lineage was promoted by culturing the cells on graphene. ${ }^{166}$ Moreover, carbon nanotubes also show great potential in cardiac regeneration, thanks to their electroactive nature. Cardiomyogenic differentiation of MSCs was enhanced when cells were seeded on carboxyl-modified, SWCNT-based PLA scaffolds, electrically stimulated, and treated with carboxyl modified SWCNTs in growth medium. ${ }^{167}$ In an in vivo study, electrospun PCL/ MWCNT/poly(acrylic acid)/poly(vinyl alcohol)(PAA/PVA) hydrogels promoted myogenic cell growth. ${ }^{168}$ In another study, PCL-thiophene conjugated CNTs were shown to support cardiomyocyte proliferation, ${ }^{169}$ and in a recent study, nanostructured CNT carpets combined with microscale aligned fibrous architecture effectively promoted multiple myocyte fusion into multinucleated myotubes. $^{170}$

\section{Drug delivery}

Due to their high surface areas and loading efficiency, 1D and 2D carbon nanomaterials can be used as highly versatile backbones for drug delivery. Most chemotherapeutic agents are antimetabolites (i.e., nucleotide analogues), cell cycle inhibitors, alkylating reagents or cytotoxic molecules, all of which are small molecules with hydrophobic properties. This enables them to interact with carbon nanomaterials through strong $\pi-\pi$ interactions, which are only reversed through an external stimulus such as $\mathrm{pH}$ change.

There is substantial research on the drug loading and release behaviour of carbon nanomaterials. Panczyk et al. has computationally shown that the chemotherapeutic drug doxorubicin (DOX) and several other hydrophobic dye molecules are readily adsorbed onto SWCNTs, and due to the protonation of the molecules at lower $\mathrm{pH}$ values, their release becomes favourable in low-pH environments that characterize tumor niches. ${ }^{171}$ Exploiting this behaviour, Depan et al. has shown that both DOX and folate-chitosan (a targeting molecule) can be loaded on GO sheets simply by mixing the two components with GO dispersed in aqueous solution. ${ }^{172}$ DOX was released slowly in $\mathrm{pH} 5.3$, but was retained in physiological pH 7.4. More recently, Singh et al. devised a Paclitaxel (PTX) loaded system where they conjugated riboflavin and thiamine on amine-functionalized MWCNTs. ${ }^{173}$ This enabled selective targeting of MCF-7 cells in conjunction with the $\mathrm{pH}$-dependent release of PTX. Gao et al. proposed a simple crosslinking-based method to functionalize GO sheets with PEGMA, which didn't interfere with the drug adsorption capacity via $\pi-\pi$ interactions and still permitted drug release in low-pH environments. ${ }^{174}$ Fedeli et al. produced MWCNTs functionalized with azido groups to make them compatible with click reactions. They then clicked biotin for the selective internalization of CNTs, BODIPY for imaging, and adsorbed DOX for cytotoxicity. ${ }^{175}$ Cao et al. used oxidized MWCNTs and decorated them with $\mathrm{Fe}_{3} \mathrm{O}_{4}$ nanoparticles to obtain magnetized MWCNTs, and loaded them with chemotherapeutic chelerythrine for targeted drug delivery. ${ }^{176}$ Liu et al. used simple starch both as a reducing agent and as a functionalization molecule to obtain water-soluble graphene sheets in a facile one-pot 
A

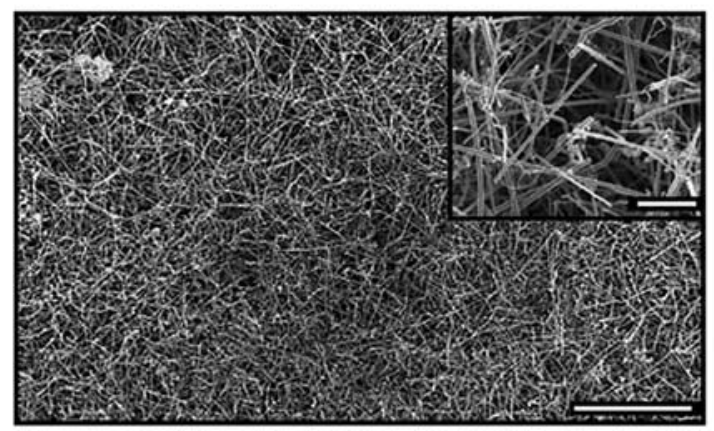

B

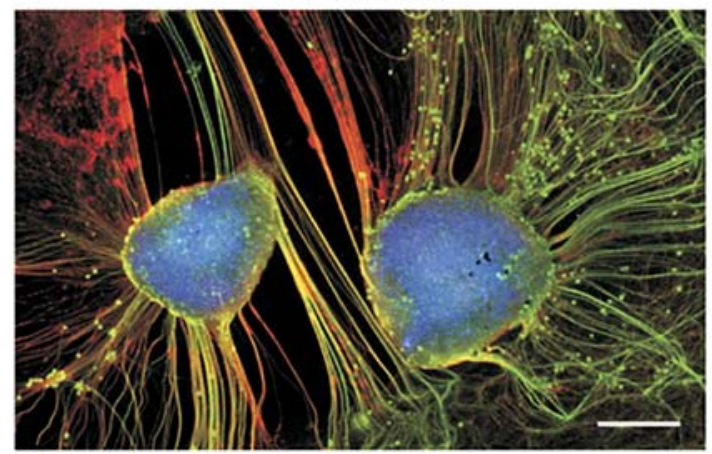

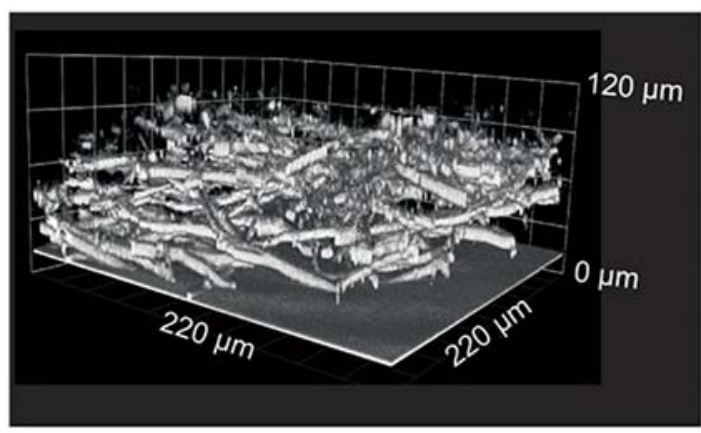

3D CNF

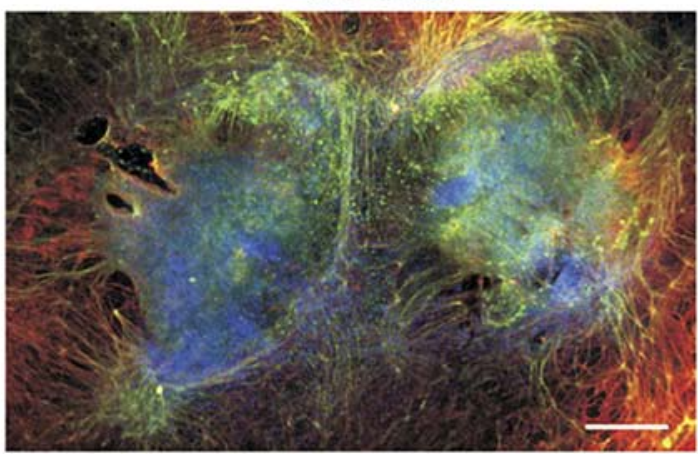

Figure 7. (A) SEM micrographs (left) and confocal 3D reconstruction (right) of MWCNT meshes. (B) Spinal organotypic slices cultured in control and 3D CNF (carbon nanotube frame) at day 14 (BIII tubulin: red, neurofilament H: green, Nuclei (DAPI): blue). Reprinted with permission from AAAS. ${ }^{161}$ Copyright (2016).

synthesis method. ${ }^{177}$ They then used this material to load hydroxycamptothectin, a chemotherapeutic that can be released in acidic $\mathrm{pH}$ to cause cytotoxicity in the tumor microenvironment.

Another important property of the carbon nanomaterials is the photothermal effect. When excited at near-IR wavelengths, they exhibit hyperthermia, experiencing a rapid increase of temperature (up to $40{ }^{\circ} \mathrm{C}$ ) ${ }^{178}$ to induce targeted and controlled cytotoxicity. This property allows carbon nanomaterials to be utilized for photodynamic therapy as well. Han et al. used SWCNTs functionalized with phenoxylated dextran to this purpose. The phenoxy-group enabled $\pi-\pi$ interactions with SWCNTs, while dextran facilitated the selective targeting of macrophages. Temperature increases from $20{ }^{\circ} \mathrm{C}$ to $60{ }^{\circ} \mathrm{C}$ were recorded following near IR irradiation, allowing the material to eliminate specific cells without damaging the surrounding tissue. Similarly, Sharker et al. utilized the $\mathrm{pH}$ responsive polymer poly(2-dimethyl amino ethyl methacrylate) (PDMEAEMA) to fabricate GO nanosheets that exert photothermal activity only at acidic $\mathrm{pH}^{179}$ Battogtokh et al. also rendered $\mathrm{GO}$ sheets $\mathrm{pH}$-sensitive by adding an aconityl-linked folate-pheophorbide A complex, folate (for tumor targeting) and pheophorbide A (as a photosensitizer), further improving GO's innate photothermal capabilities. $^{180}$

There are many other strategies and applications that utilize carbon nanomaterials as drug delivery platforms. For example, de Souza et al. showed that the adsorption of cisplatin into
SWCNTs is kinetically favorable, and that carbon nanomaterials can be used to deliver inorganic drugs to specific sites as well. ${ }^{181}$ Anderson et al. fabricated PEGylated SWCNTs, functionalized them with poly(allylamine hydrochloride), and adsorbed siRNAs on them to produce a CNT-based transfection agent. ${ }^{182}$ Similarly, Siu et al. has modified SWCNTs with lipid-modified poly(ethyleneimine) for successful siRNA delivery both in vitro and in vivo. ${ }^{183}$ Weaver et al. proposed a novel polypyrrole-GO-dexamethasone complex that can release the anti-inflammatory drug dexamethasone upon electrical stimulation. ${ }^{184}$ Mo et al. described an aptamer-based assembly, where ATP binding to the aptamers would disrupt the assembly and release the adsorbed DOX. ${ }^{185}$ de Sousa et al. thiolated GO sheets in order to increase their mucoadhesive properties and potentially use the hydrophobic drug adsorbed to the sheets as an oral delivery agent. ${ }^{186}$ Last but not least, Singh et al. conjugated functional superoxide dismutase enzymes onto MWCNTs to alleviate oxidative stress, as a model for enzyme delivery with carbon nanomaterials. ${ }^{187}$

\section{Bioimaging}

Carbon nanomaterials, especially when modified through the above-mentioned methods, show desirable biocompatible characteristics, such as being soluble in aqueous media, low cytotoxicity, and biostability. In addition, they provide a means to functionalize the designed system with various other moieties, 


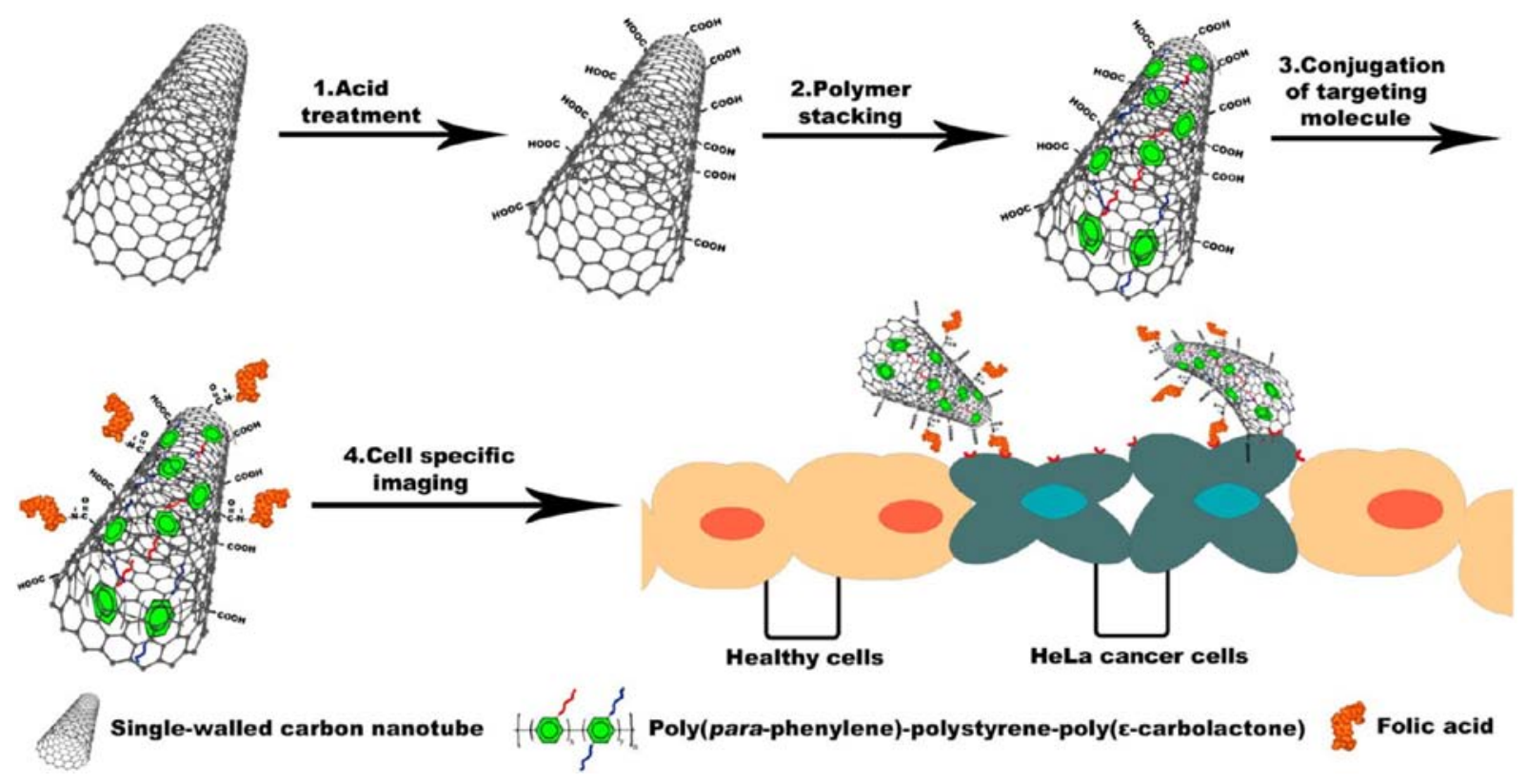

Figure 8. Schematic representation of conjugation process and cell specific imaging. Reprinted (adapted) with permission from American Chemical Society. ${ }^{190}$ Copyright (2013).

such as drugs, targeting molecules, and fluorescent or magnetic probes. Carbon dots, or graphene quantum dots (QDs), are the most commonly used carbon nanomaterials for bioimaging and show excellent fluorescent characteristics. However, 0D materials are out of the scope of this review and will not be covered any further. Comprehensive reviews on the subject have been provided by Shi et al. ${ }^{188}$ or Wang et al. ${ }^{189}$

Although carbon nanomaterials can utilize the innate fluorescent ability of the materials for bioimaging applications, most research focuses on their use as a backbone conjugated with magnetic or fluorescent moieties. Ag et al., for example, noncovalently functionalized SWCNTs with a fluorescent copolymer, poly(para-phenylene), with polystyrene and PCL side chains to obtain fluorescent SWCNT probes. ${ }^{190}$ They then conjugated folic acid to target the HeLa cells in culture (Figure 8). In addition, $\mathrm{Hu}$ et al. demonstrated that SWCNTs functionalized with a fluorescent naphthalenediimide moiety show good solvability in both organic and aqueous solvents, are biocompatible, and can be functionalized further through secondary reactions. ${ }^{191}$ Khandare et al. reported a highly versatile nanoprobe; composed of a MWCNT backbone that is functionalized with FITC for fluorescence, $\mathrm{Fe}_{3} \mathrm{O}_{4}$ for magnetization, and PEG for biocompatibility and aqueous stability, as a multifunctional bioimaging material. ${ }^{192}$ Kundu et al. reported that covalent attachment of poly(N-isopropylacrylamide) onto GO sheets added both fluorescence and the capacity for the $\mathrm{pH}$-dependent release of both hydrophobic and hydrophilic drugs to the structure. ${ }^{193}$ Liu et al. developed a method in which MWCNTs are filled with magnetic nanoparticles and rendered water-soluble by the addition of amine groups onto the surface of the tubes, producing a composite nanomaterial that can be used as a MRI contrast agent or in photodynamic therapeutics. ${ }^{194}$ Shi et al. decorated GO sheets with both iron oxide and gold nanoparticles, PEGylated them for biocompatibility and aqueous stability, and proposed their use as photodynamic therapeutics and tools for magnetic bioimaging. ${ }^{195}$ Finally, Wate et al. reported the synthesis of multifunctional GO probes functionalized covalently with a poly(amido amine) dendrimer containing $\mathrm{Fe}_{3} \mathrm{O}_{4}$, and $\mathrm{Cy} 5$ to obtain a magnetically responsive, fluorescent probe with enhanced biostability. ${ }^{196}$

\section{Biosensors}

Biosensors are used to analyze or detect analytes with the use of a biological component. The addition of biological components to biosensor systems aims to increase the sensitivity, accuracy and sustainability of sensor systems. Carbon nanomaterials have been used as scaffolds for biosensing applications due to their fluorescence quenching capabilities. Based on fluorescent resonance energy transfer (FRET), the change in fluorescent intensity can be used to measure the amount of the analyte. For example, on a system designed by Tian et al. ${ }^{197}$ a fluorophore was immobilized on GO sheets with a caspase-3 specific peptide amphiphile spacer through noncovalent interactions. When caspase activity was absent, fluorescence of the fluorophore would be quenched by GO, but fluorescence could be observed in the presence of caspase. Similarly, Dong et al. ${ }^{198}$ have designed a QD-GO coupled system using an aptamer-based molecular beacon probe as a spacer (Figure 9). The binding of an analyte to the probe would increase the distance between the QD and $\mathrm{GO}$, which would lead to an increase in fluorescence intensity directly correlated to the amount of analyte. Similarly, $\mathrm{Lu}$ et al. has adsorbed dye-labeled single stranded DNA probes onto GO sheets, which are quenched when in close contact with the surface. ${ }^{199}$ However, when a sequence complimentary to the adsorbed probe is introduced, hybridization between the two strands would free the probe and fluorescence would be restored. 


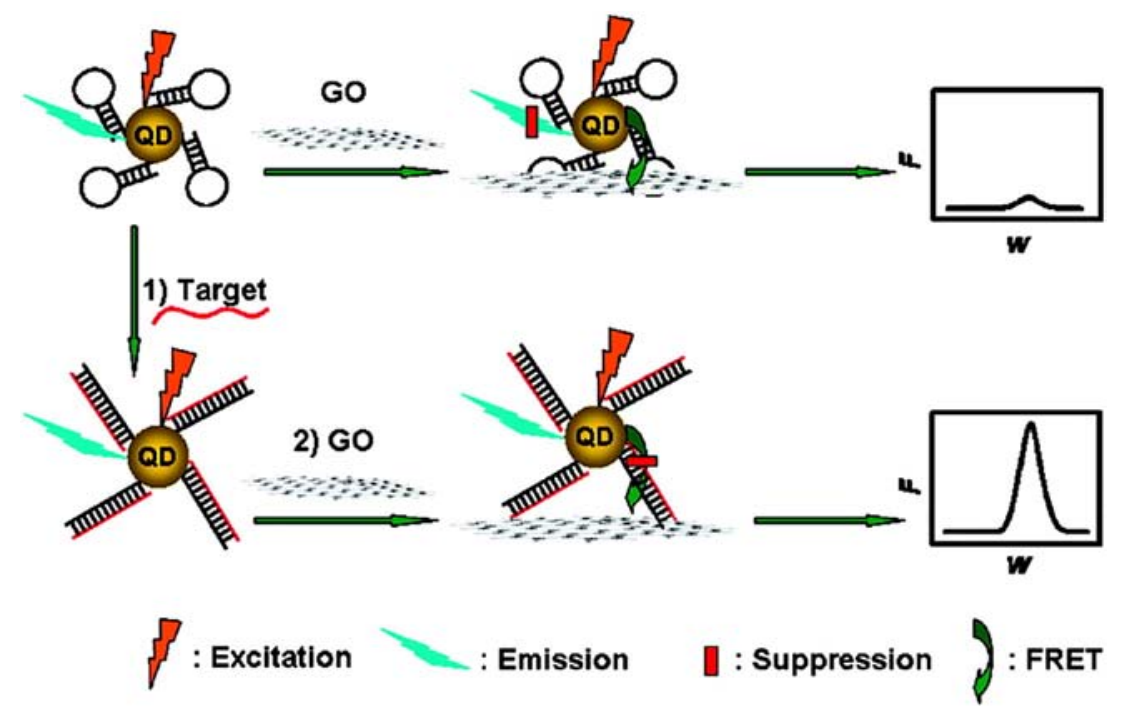

Figure 9. Schematic representation of GO-induced fluorescence quenching of molecular beacon-QDs and biosensing mechanism. Reprinted (adapted) with permission from American Chemical Society. ${ }^{198}$ Copyright (2010).

Another feature used in biosensing applications is the electrical conductivity of carbon nanomaterials, matched with electrochemical reactions, i.e., redox reactions. Enzyme-mediated reduction or oxidation reactions would produce electrons, and this would result in a measurable potentiometric or amperometric difference in the biosensor system. Carbon nanomaterials can be used as electrodes themselves in biosensor systems, or merely as electrode functionalization materials. In the work by Barsan et al., conventional carbon film electrodes were first functionalized with electroactive polymers poly(brilliant green) and PEDOT, followed by CNT adsorption onto the surfaces. ${ }^{200}$ Glucose oxidase, alcohol oxidase and alcohol dehydrogenase were used as model enzymes, and for both analytes, limits of detection comparable to those found in the literature were obtained using this novel CNT-based biosensor.

\section{Conclusions and future perspectives}

The 1D and 2D carbon nanomaterials have received considerable interest in the biomedical field recently due to their unique structures and intrinsic properties. In this review, we have described the synthesis, functionalization and applications of CNT, graphene and their derivatives, with emphasis on their biocompatibility and potential utility in medicine. Various functionalization methods, including covalent and noncovalent types, offer useful approaches to expand the range of tasks that $1 \mathrm{D}$ and 2D carbon-nanostructures are able to accomplish. In particular, the conjugation of functional moieties such as drugs, targeting molecules, and fluorescent or magnetic probes to 1D and $2 \mathrm{D}$ carbon-nanostructures effectively transforms them into bioactive agents that combine their inherent mechanical, electrical and optical properties with the ability to directly interfere with biological signaling networks. The absorption and emission spectra of these composite materials, for example, are ideal for in vivo targeted tissue imaging, while their mechanical integrity, ultralow density, unique nanotopography and intrinsic conductivity makes them strong candidates for next-generation tissue engineering scaffolds. In addition, multiple types of functional moieties can be attached to a single nanomaterial platform, creating a multifunctional material that is able to perform a broad range of roles while circumventing the toxicity issues associated with $1 \mathrm{D}$ and 2D carbon-nanostructures. An ideal system should compromise between sufficient graphene, $\mathrm{GO}$ or CNT concentration and minimum or no toxicity. Current results suggest that ROS production is the most important cytotoxic mechanism of these nanostructures. Besides, the synthesis and functionalization type, purity, exposure route, time and dosage play huge roles on the cytotoxicity of $1 \mathrm{D}$ and $2 \mathrm{D}$ carbon-nanostructures. Depending on the functionalization type, these materials can be tailored, together with their biocompatibility, to increase their retention time in the body in order to be used in drug delivery or cancer therapy purposes. However, an overall conclusion about the toxicity of $1 \mathrm{D}$ and $2 \mathrm{D}$ carbon-nanostructures cannot be clearly drawn due to the aforementioned variable parameters and lack of nonsystematic investigations of $1 \mathrm{D}$ and 2D carbon-nanostructures. However, it should be stressed that low batch-to-batch variances in size, shape, morphology and surface characteristics are necessary for the effective use of $1 \mathrm{D}$ and $2 \mathrm{D}$ carbon nanomaterials in biomedical settings, as small changes in material properties may result in large changes in biological function. In addition, greater attention should be paid to the biosafety of these materials, especially with regards to their long term toxicity, cellular-uptake mechanisms and in vivo metabolic pathways.

\section{Acknowledgements}

We thank to Alper Devrim Özkan for his fruitful discussions and his comments on the manuscript. This work is partially supported by TUBITAK and TUBA. 


\section{References}

1. Kroto HW, Heath JR, O’Brien SC, Curl RF, Smalley RE. C 60: buckminsterfullerene. Nature 1985;318(6042):162-3.

2. Iijima S. Helical microtubules of graphitic carbon. Nature 1991;354(6348):56-8.

3. Novoselov KS, Geim AK, Morozov SV, Jiang D, Zhang Y, Dubonos $\mathrm{SV}$, et al. Electric field effect in atomically thin carbon films. Science 2004;306(5696):666-9.

4. Gao Z, Varela JA, Groc L, Lounis B, Cognet L. Toward the suppression of cellular toxicity from single-walled carbon nanotubes. Biomater Sci 2016;4(2):230-44.

5. Hong G, Diao S, Antaris AL, Dai H. Carbon nanomaterials for biological imaging and nanomedicinal therapy. Chem Rev 2015;115(19):10816-906.

6. Harrison BS, Atala A. Carbon nanotube applications for tissue engineering. Biomaterials 2007;28(2):344-53.

7. Yang Y, Asiri AM, Tang Z, Du D, Lin Y. Graphene based materials for biomedical applications. Mater Today 2013;16(10):365-73.

8. Yang Z, Ren J, Zhang Z, Chen X, Guan G, Qiu L, et al. Recent advancement of nanostructured carbon for energy applications. Chem Rev 2015;115(11):5159-223.

9. Liu Y, Kumar S. Polymer/carbon nanotube nano composite fibers - a review. ACS Appl Mater Interfaces 2014;6(9):6069-87.

10. Yang N, Chen X, Ren T, Zhang P, Yang D. Carbon nanotube based biosensors. Sens Actuators B 2015;207:690-715.

11. Miyauchi Y. Photoluminescence studies on exciton photophysics in carbon nanotubes. J Mater Chem C 2013;1(40):6499-521.

12. Wang H, Ma H, Zheng W, An D, Na C. Multifunctional and recollectable carbon nanotube ponytails for water purification. ACS Appl Mater Interfaces 2014;6(12):9426-34.

13. Alshehri R, Ilyas AM, Hasan A, Arnaout A, Ahmed F, Memic A. Carbon nanotubes in biomedical applications: Factors, mechanisms and remedies of toxicity. J Med Chem 2016;59(18):8149-67, http:// dx.doi.org/10.1021/acs.jmedchem.5b01770.

14. Bacon R. Growth, structure, and properties of graphite whiskers. J Appl Phys 1960;31(2):283-90.

15. Zhang D-B, Akatyeva E, Dumitrică T. Bending ultrathin graphene at the margins of continuum mechanics. Phys Rev Lett 2011;106(25):255503.

16. Bekyarova E, Ni Y, Malarkey EB, Montana V, McWilliams JL, Haddon RC, et al. Applications of carbon nanotubes in biotechnology and biomedicine. J Biomed Nanotechnol 2005;1(1):3-17.

17. Odom TW, Huang J-L, Kim P, Lieber CM. Structure and electronic properties of carbon nanotubes. J Phys Chem B 2000;104(13):2794-809.

18. Sgobba V, Ehli C, Guldi DM. Covalent approaches towards multifunctional carbon-nanotube materials. In: Langa F, Nierengarten JF, editors. Fullerenes: Principles and Applications. 20: RSC Nanoscience \& Nanotechnology; 2011. p. 549-612.

19. Han ZJ, Rider AE, Ishaq M, Kumar S, Kondyurin A, Bilek MM, et al. Carbon nanostructures for hard tissue engineering. RSC Adv 2013;3(28):11058-72.

20. Pop E, Mann D, Wang Q, Goodson K, Dai H. Thermal conductance of an individual single-wall carbon nanotube above room temperature. Nano Lett 2006;6(1):96-100.

21. Liu Z, Tabakman S, Welsher K, Dai H. Carbon nanotubes in biology and medicine: in vitro and in vivo detection, imaging and drug delivery. Nano Res 2009;2(2):85-120.

22. Shi Z, Lian Y, Zhou X, Gu Z, Zhang Y, Iijima S, et al. Mass-production of single-wall carbon nanotubes by arc discharge method. Carbon 1999;37(9):1449-53.

23. Hinkov I, Farhat S, Scott C. Influence of the gas pressure on single-wall carbon nanotube formation. Carbon 2005;43(12):2453-62.

24. Journet C, Maser W, Bernier P, Loiseau A, De La Chapelle ML, Lefrant $\mathrm{d} 1 \mathrm{~S}$, et al. Large-scale production of single-walled carbon nanotubes by the electric-arc technique. Nature 1997;388(6644):756-8.
25. Guo T, Nikolaev P, Thess A, Colbert D, Smalley R. Catalytic growth of single-walled manotubes by laser vaporization. Chem Phys Lett 1995;243(1):49-54.

26. Baker R. Catalytic growth of carbon filaments. Carbon 1989;27(3):315-23.

27. Yan Y, Miao J, Yang Z, Xiao F-X, Yang HB, Liu B, et al. Carbon nanotube catalysts: recent advances in synthesis, characterization and applications. Chem Soc Rev 2015;44(10):3295-346.

28. Bronikowski MJ, Willis PA, Colbert DT, Smith K, Smalley RE. Gasphase production of carbon single-walled nanotubes from carbon monoxide via the HiPco process: A parametric study. $J$ Vacuum Sci Technol A 2001;19(4):1800-5.

29. Park T-J, Banerjee S, Hemraj-Benny T, Wong SS. Purification strategies and purity visualization techniques for single-walled carbon nanotubes. J Mater Chem 2006;16(2):141-54.

30. Datsyuk V, Kalyva M, Papagelis K, Parthenios J, Tasis D, Siokou A, et al. Chemical oxidation of multiwalled carbon nanotubes. Carbon 2008;46(6):833-40.

31. Ko C-J, Lee C-Y, Ko F-H, Chen H-L, Chu T-C. Highly efficient microwave-assisted purification of multiwalled carbon nanotubes. Microelectron Eng 2004;73:570-7.

32. Novoselov KS, Fal V, Colombo L, Gellert P, Schwab M, Kim K. A roadmap for graphene. Nature 2012;490(7419):192-200.

33. Kuila T, Bose S, Mishra AK, Khanra P, Kim NH, Lee JH. Chemical functionalization of graphene and its applications. Prog Mater Sci 2012;57(7):1061-105.

34. Jastrzębska AM, Kurtycz P, Olszyna AR. Recent advances in graphene family materials toxicity investigations. J Nanopart Res 2012;14(12):1320.

35. Ray SC. Application and uses of graphene oxide and reduced graphene oxide. Applications of Graphene and Graphene-Oxide Based Nanomaterials. Oxford: William Andrew Publishing; 2015. p. 39-55.

36. Pandey D, Reifenberger R, Piner R. Scanning probe microscopy study of exfoliated oxidized graphene sheets. Surf Sci 2008;602(9):1607-13.

37. Balandin AA. Thermal properties of graphene and nanostructured carbon materials. Nat Mater 2011;10(8):569-81.

38. Dragoman D, Dragoman M. Transport properties of carbon nanotubes and graphene. Carbon nanotubes and graphene. 2nd edn. Elsevier; 2014. p. 151-64.

39. Sanchez VC, Jachak A, Hurt RH, Kane AB. Biological interactions of graphene-family nanomaterials: an interdisciplinary review. Chem Res Toxicol 2011;25(1):15-34.

40. Edwards RS, Coleman KS. Graphene synthesis: relationship to applications. Nanoscale 2013;5(1):38-51.

41. Park S, Ruoff RS. Chemical methods for the production of graphenes. Nat Nanotechnol 2009;4(4):217-24.

42. Xia ZY, Pezzini S, Treossi E, Giambastiani G, Corticelli F, Morandi V, et al. The exfoliation of graphene in liquids by electrochemical, chemical, and sonication-assisted techniques: A nanoscale study. Adv Funct Mater 2013;23(37):4684-93.

43. Hernandez Y, Nicolosi V, Lotya M, Blighe FM, Sun Z, De S, et al. High-yield production of graphene by liquid-phase exfoliation of graphite. Nat Nanotechnol 2008;3(9):563-8.

44. Jiao L, Zhang L, Wang X, Diankov G, Dai H. Narrow graphene nanoribbons from carbon nanotubes. Nature 2009;458(7240):877-80.

45. Li D, Mueller MB, Gilje S, Kaner RB, Wallace GG. Processable aqueous dispersions of graphene nanosheets. Nat Nanotechnol 2008;3(2):101-5.

46. Li X, Cai W, An J, Kim S, Nah J, Yang D, et al. Large-area synthesis of high-quality and uniform graphene films on copper foils. Science 2009;324(5932):1312-4.

47. Van Noorden R. Production: Beyond sticky tape. Nature 2012;483(7389):S32-3.

48. Mao HY, Laurent S, Chen W, Akhavan O, Imani M, Ashkarran AA, et al. Graphene: promises, facts, opportunities, and challenges in nanomedicine. Chem Rev 2013;113(5):3407-24.

49. Eigler S, Hirsch A. Chemistry with graphene and graphene oxide challenges for synthetic chemists. Angew Chem Int Ed 2014;53(30):7720-38. 
50. Skoda M, Dudek I, Jarosz A, Szukiewicz D. Graphene: one material, many possibilities - application difficulties in biological systems. $J$ Nanomater 2014;2014:190.

51. Hummers Jr WS, Offeman RE. Preparation of graphitic oxide. J Am Chem Soc 1958;80(6) [1339-].

52. Cooper DR, D’Anjou B, Ghattamaneni N, Harack B, Hilke M, Horth A, et al. Experimental review of graphene. ISRN Condensed Matter Phys 2012;2012.

53. Norimatsu W, Kusunoki M. Epitaxial graphene on $\operatorname{SiC}\{0001\}$ : advances and perspectives. Phys Chem Chem Phys 2014;16(8):3501-11.

54. Soldano C, Mahmood A, Dujardin E. Production, properties and potential of graphene. Carbon 2010;48(8):2127-50.

55. Kuilla T, Bhadra S, Yao D, Kim NH, Bose S, Lee JH. Recent advances in graphene based polymer composites. Prog Polym Sci 2010;35(11):1350-75.

56. Bae S, Kim H, Lee Y, Xu X, Park J-S, Zheng Y, et al. Roll-to-roll production of 30-inch graphene films for transparent electrodes. Nat Nanotechnol 2010;5(8):574-8.

57. Liu Z, Tabakman SM, Chen Z, Dai H. Preparation of carbon nanotube bioconjugates for biomedical applications. Nat Protoc 2009;4(9):1372-81.

58. Zhao J, Balbuena PB. Structural and reactivity properties of finite length cap-ended single-wall carbon nanotubes. Eur J 2006;110(8):2771-5.

59. Charlier J-C. Defects in carbon nanotubes. Acc Chem Res 2002;35(12):1063-9.

60. Battigelli A, Ménard-Moyon C, Da Ros T, Prato M, Bianco A. Endowing carbon nanotubes with biological and biomedical properties by chemical modifications. Adv Drug Deliv Rev 2013;65(15):1899-920.

61. Dujardin E, Ebbesen TW, Krishnan A, Treacy MM. Purification of single-shell nanotubes. Adv Mater 1998;10(8):611-3.

62. Ovejero G, Sotelo J, Romero M, Rodríguez A, Ocana M, Rodríguez G, et al. Multiwalled carbon nanotubes for liquid-phase oxidation. Functionalization, characterization, and catalytic activity. Ind Eng Chem Res 2006; 45(7):2206-12.

63. Hernadi K, Siska A, Thien-Nga L, Forro L, Kiricsi I. Reactivity of different kinds of carbon during oxidative purification of catalytically prepared carbon nanotubes. Solid State Ion 2001;141:203-9.

64. Georgakilas V, Kordatos K, Prato M, Guldi DM, Holzinger M, Hirsch A. Organic functionalization of carbon nanotubes. $J$ Am Chem Soc 2002;124(5):760-1.

65. Villa CH, Dao T, Ahearn I, Fehrenbacher N, Casey E, Rey DA, et al. Single-walled carbon nanotubes deliver peptide antigen into dendritic cells and enhance IgG responses to tumor-associated antigens. ACS Nano 2011;5(7):5300-11.

66. Wu W, Wieckowski S, Pastorin G, Benincasa M, Klumpp C, Briand JP, et al. Targeted delivery of amphotericin B to cells by using functionalized carbon nanotubes. Angew Chem Int Ed 2005;44(39):6358-62.

67. Dumortier H, Lacotte S, Pastorin G, Marega R, Wu W, Bonifazi D, et al. Functionalized carbon nanotubes are non-cytotoxic and preserve the functionality of primary immune cells. Nano Lett 2006;6(7):1522-8.

68. Han J, Gao C. Functionalization of carbon nanotubes and other nanocarbons by azide chemistry. Nano Micro Lett 2010;2(3):213-26.

69. Chen Y, Haddon R, Fang S, Rao AM, Eklund P, Lee W, et al. Chemical attachment of organic functional groups to single-walled carbon nanotube material. J Mater Res 1998;13(09):2423-31.

70. Wu H-C, Chang X, Liu L, Zhao F, Zhao Y. Chemistry of carbon nanotubes in biomedical applications. J Mater Chem 2010;20(6):1036-52.

71. Sgobba V, Rahman GA, Ehli C, Guldi DM. Covalent and non-covalent approaches towards multifunctional carbon nanotube materials. In: Langa F, Nierengarten JF, editors. Fullerenes: Principles and Applications: RSC Nanoscience \& Nanotechnology; 2007. p. 329-79.

72. Moore VC, Strano MS, Haroz EH, Hauge RH, Smalley RE, Schmidt J, et al. Individually suspended single-walled carbon nanotubes in various surfactants. Nano Lett 2003;3(10):1379-82.

73. Paloniemi H, Ääritalo T, Laiho T, Liuke H, Kocharova N, Haapakka K, et al. Water-soluble full-length single-wall carbon nanotube polyelectrolytes: preparation and characterization. J Phys Chem B 2005;109(18):8634-42.
74. Karousis N, Tagmatarchis N, Tasis D. Current progress on the chemical modification of carbon nanotubes. Chem Rev 2010;110(9):5366-97.

75. Liu Z, Yang K, Lee S-T. Single-walled carbon nanotubes in biomedical imaging. J Mater Chem 2011;21(3):586-98.

76. Sheikholeslam M, Pritzker M, Chen P. Dispersion of multiwalled carbon nanotubes in water using ionic-complementary peptides. Langmuir 2012;28(34):12550-6.

77. Wang S, Humphreys ES, Chung S-Y, Delduco DF, Lustig SR, Wang $\mathrm{H}$, et al. Peptides with selective affinity for carbon nanotubes. Nat Mater 2003;2(3):196-200.

78. Dieckmann GR, Dalton AB, Johnson PA, Razal J, Chen J, Giordano $\mathrm{GM}$, et al. Controlled assembly of carbon nanotubes by designed amphiphilic peptide helices. $J$ Am Chem Soc 2003;125(7):1770-7.

79. Arnold MS, Guler MO, Hersam MC, Stupp SI. Encapsulation of carbon nanotubes by self-assembling peptide amphiphiles. Langmuir 2005;21(10):4705-9.

80. Chen RJ, Zhang Y, Wang D, Dai H. Noncovalent sidewall functionalization of single-walled carbon nanotubes for protein immobilization. J Am Chem Soc 2001;123(16):3838-9.

81. Marega R, Bonifazi D. Filling carbon nanotubes for nanobiotechnological applications. Chem 2014;38(1):22-7.

82. Bourlinos AB, Gournis D, Petridis D, Szabó T, Szeri A, Dékány I. Graphite oxide: chemical reduction to graphite and surface modification with primary aliphatic amines and amino acids. Langmuir 2003;19(15):6050-5.

83. Xu LQ, Yang WJ, Neoh K-G, Kang E-T, Fu GD. Dopamine-induced reduction and functionalization of graphene oxide nanosheets. Macromolecules 2010;43(20):8336-9.

84. Pham TA, Kumar NA, Jeong YT. Covalent functionalization of graphene oxide with polyglycerol and their use as templates for anchoring magnetic nanoparticles. Synth Met 2010;160(17):2028-36.

85. Liu Z, Robinson JT, Sun X, Dai H. PEGylated nanographene oxide for delivery of water-insoluble cancer drugs. J Am Chem Soc 2008;130(33):10876-7.

86. Stankovich S, Piner RD, Chen X, Wu N, Nguyen ST, Ruoff RS. Stable aqueous dispersions of graphitic nanoplatelets via the reduction of exfoliated graphite oxide in the presence of poly (sodium 4styrenesulfonate). J Mater Chem 2006;16(2):155-8.

87. Xu Y, Bai H, Lu G, Li C, Shi G. Flexible graphene films via the filtration of water-soluble noncovalent functionalized graphene sheets. J Am Chem Soc 2008;130(18):5856-7.

88. Choi E-Y, Han TH, Hong J, Kim JE, Lee SH, Kim HW, et al. Noncovalent functionalization of graphene with end-functional polymers. J Mater Chem 2010;20(10):1907-12.

89. Liu J, Yang W, Tao L, Li D, Boyer C, Davis TP. Thermosensitive graphene nanocomposites formed using pyrene-terminal polymers made by RAFT polymerization. Polym Chem 2010;48(2):425-33.

90. Yang H, Zhang Q, Shan C, Li F, Han D, Niu L. Stable, conductive supramolecular composite of graphene sheets with conjugated polyelectrolyte. Langmuir 2010;26(9):6708-12.

91. Sajid MI, Jamshaid U, Jamshaid T, Zafar N, Fessi H, Elaissari A. Carbon nanotubes from synthesis to in vivo biomedical applications. Pharm 2016;501(1):278-99.

92. Zhu Y, Murali S, Stoller MD, Ganesh K, Cai W, Ferreira PJ, et al. Carbon-based supercapacitors produced by activation of graphene. Science 2011;332(6037):1537-41.

93. Zhang Y, Brar VW, Girit C, Zettl A, Crommie MF. Origin of spatial charge inhomogeneity in graphene. Nat Phys 2009;5(10):722-6.

94. Teague M, Lai A, Velasco J, Hughes C, Beyer A, Bockrath M, et al. Evidence for strain-induced local conductance modulations in singlelayer graphene on SiO2. Nano Lett 2009;9(7):2542-6.

95. Reddy C, Rajendran S, Liew K. Equilibrium configuration and continuum elastic properties of finite sized graphene. Nanotechnology 2006;17(3):864.

96. Lee C, Wei X, Kysar JW, Hone J. Measurement of the elastic properties and intrinsic strength of monolayer graphene. Science 2008;321(5887):385-8. 
97. Dikin DA, Stankovich S, Zimney EJ, Piner RD, Dommett GH, Evmenenko G, et al. Preparation and characterization of graphene oxide paper. Nature 2007;448(7152):457-60.

98. Dresselhaus MS, Jorio A, Hofmann M, Dresselhaus G, Saito R. Perspectives on carbon nanotubes and graphene Raman spectroscopy. Nano Lett 2010;10(3):751-8.

99. Ferrari A, Meyer J, Scardaci V, Casiraghi C, Lazzeri M, Mauri F, et al. Raman spectrum of graphene and graphene layers. Phys Rev Lett 2006;97(18):187401.

100. Dyke CA, Tour JM. Solvent-free functionalization of carbon nanotubes. J Am Chem Soc 2003;125(5):1156-7.

101. Gurunathan S, Kim J-H. Synthesis, toxicity, biocompatibility, and biomedical applications of graphene and graphene-related materials. Nanomedicine 2016;11:1927.

102. Bussy C, Ali-Boucetta H, Kostarelos K. Safety considerations for graphene: lessons learnt from carbon nanotubes. Acc Chem Res 2012;46(3):692-701.

103. Linares J, Matesanz MCn, Vila M, Feito MJ, Gonçalves G, Vallet-Regí $\mathrm{M}$, et al. Endocytic mechanisms of graphene oxide nanosheets in osteoblasts, hepatocytes and macrophages. ACS Appl Mater Interfaces 2014;6(16):13697-706.

104. Zhang Y, Ali SF, Dervishi E, Xu Y, Li Z, Casciano D, et al. Cytotoxicity effects of graphene and single-wall carbon nanotubes in neural phaeochromocytoma-derived PC12 cells. ACS Nano 2010;4(6):3181-6.

105. Sasidharan A, Panchakarla L, Chandran P, Menon D, Nair S, Rao C, et al. Differential nano-bio interactions and toxicity effects of pristine versus functionalized graphene. Nanoscale 2011;3(6):2461-4.

106. Lammel T, Boisseaux P, Fernández-Cruz M-L, Navas JM. Internalization and cytotoxicity of graphene oxide and carboxyl graphene nanoplatelets in the human hepatocellular carcinoma cell line Hep G2. Part Fibre Toxicol 2013;10(1):1.

107. Chang Y, Yang S-T, Liu J-H, Dong E, Wang Y, Cao A, et al. In vitro toxicity evaluation of graphene oxide on A549 cells. Toxicol Lett 2011;200(3):201-10.

108. Qu G, Liu S, Zhang S, Wang L, Wang X, Sun B, et al. Graphene oxide induces toll-like receptor 4 (TLR4)-dependent necrosis in macrophages. ACS Nano 2013;7(7):5732-45.

109. Liu C-W, Xiong F, Jia H-Z, Wang X-L, Cheng H, Sun Y-H, et al. Graphene-based anticancer nanosystem and its biosafety evaluation using a zebrafish model. Biomacromolecules 2013;14(2):358-66.

110. Duch MC, Budinger GS, Liang YT, Soberanes S, Urich D, Chiarella $\mathrm{SE}$, et al. Minimizing oxidation and stable nanoscale dispersion improves the biocompatibility of graphene in the lung. Nano Lett 2011;11(12):5201-7.

111. Yang K, Wan J, Zhang S, Zhang Y, Lee S-T, Liu Z. In vivo pharmacokinetics, long-term biodistribution, and toxicology of PEGylated graphene in mice. ACS Nano 2010;5(1):516-22.

112. Kostarelos K, Lacerda L, Pastorin G, Wu W, Wieckowski S, Luangsivilay $\mathrm{J}$, et al. Cellular uptake of functionalized carbon nanotubes is independent of functional group and cell type. Nat Nanotechnol 2007;2(2):108-13.

113. Zhang Y, Xu Y, Li Z, Chen T, Lantz SM, Howard PC, et al. Mechanistic toxicity evaluation of uncoated and PEGylated singlewalled carbon nanotubes in neuronal $\mathrm{PC} 12$ cells. ACS Nano 2011;5(9):7020-33.

114. Singh SK, Singh MK, Kulkarni PP, Sonkar VK, Grácio JJ, Dash D. Amine-modified graphene: thrombo-protective safer alternative to graphene oxide for biomedical applications. ACS Nano 2012;6(3):2731-40.

115. Qiao Y, An J, Ma L. Single cell array based assay for in vitro genotoxicity study of nanomaterials. Anal Chem 2013;85(8):4107-12.

116. Wang A, Pu K, Dong B, Liu Y, Zhang L, Zhang Z, et al. Role of surface charge and oxidative stress in cytotoxicity and genotoxicity of graphene oxide towards human lung fibroblast cells. J Appl Toxicol 2013;33(10):1156-64.
117. Liu Y, Luo Y, Wu J, Wang Y, Yang X, Yang R, et al. Graphene oxide can induce in vitro and in vivo mutagenesis. Sci Rep 2013;3:3469.

118. Akhavan O, Ghaderi E, Akhavan A. Size-dependent genotoxicity of graphene nanoplatelets in human stem cells. Biomaterials 2012;33(32):8017-25.

119. Zhang Y, Petibone D, Xu Y, Mahmood M, Karmakar A, Casciano D, et al. Toxicity and efficacy of carbon nanotubes and graphene: the utility of carbon-based nanoparticles in nanomedicine. Drug Metab Rev 2014;46(2):232-46.

120. Poulsen SS, Jackson P, Kling K, Knudsen KB, Skaug V, Kyjovska ZO, et al. Multi-walled carbon nanotube physicochemical properties predict pulmonary inflammation and genotoxicity. Nanotoxicology 2016:1-13.

121. Girardi FA, Bruch GE, Peixoto CS, Dal Bosco L, Sahoo SK, Gonçalves $\mathrm{CO}$, et al. Toxicity of single-wall carbon nanotubes functionalized with polyethylene glycol in zebrafish (Danio rerio) embryos. J Appl Toxicol 2016.

122. Cheng L, Yang K, Shao M, Lu X, Liu Z. In vivo pharmacokinetics, longterm biodistribution and toxicology study of functionalized upconversion nanoparticles in mice. Nanomedicine 2011;6(8):1327-40.

123. Wang X, Podila R, Shannahan JH, Rao AM, Brown JM. Intravenously delivered graphene nanosheets and multiwalled carbon nanotubes induce site-specific Th2 inflammatory responses via the IL-33/ST2 axis. Nanomedicine 2013;8:1733-48.

124. Ma-Hock L, Strauss V, Treumann S, Küttler K, Wohlleben W, Hofmann T, et al. Comparative inhalation toxicity of multi-wall carbon nanotubes, graphene, graphite nanoplatelets and low surface carbon black. Part Fibre Toxicol 2013;10(1):1.

125. Zhang X, Yin J, Peng C, Hu W, Zhu Z, Li W, et al. Distribution and biocompatibility studies of graphene oxide in mice after intravenous administration. Carbon 2011;49(3):986-95.

126. Reshma S, Syama S, Mohanan P. Nano-biointeractions of PEGylated and bare reduced graphene oxide on lung alveolar epithelial cells: A comparative in vitro study. Biointerfaces 2016;140:104-16.

127. Orecchioni M, Bedognetti D, Sgarrella F, Marincola FM, Bianco A, Delogu LG. Impact of carbon nanotubes and graphene on immune cells. J Transl Med 2014;12(1):1.

128. Dong J, Porter DW, Batteli LA, Wolfarth MG, Richardson DL, Ma Q. Pathologic and molecular profiling of rapid-onset fibrosis and inflammation induced by multi-walled carbon nanotubes. Arch Toxicol 2015;89(4):621-33.

129. Murphy FA, Poland CA, Duffin R, Al-Jamal KT, Ali-Boucetta H, Nunes A, et al. Length-dependent retention of carbon nanotubes in the pleural space of mice initiates sustained inflammation and progressive fibrosis on the parietal pleura. Pathol 2011;178(6):2587-600.

130. Meng S, Rouabhia M, Zhang Z. Electrical stimulation modulates osteoblast proliferation and bone protein production through heparin-bioactivated conductive scaffolds. Bioelectromagnetics 2013;34(3):189-99.

131. Kalbacova M, Broz A, Kong J, Kalbac M. Graphene substrates promote adherence of human osteoblasts and mesenchymal stromal cells. Carbon 2010;48(15):4323-9.

132. Crowder SW, Prasai D, Rath R, Balikov DA, Bae H, Bolotin KI, et al. Three-dimensional graphene foams promote osteogenic differentiation of human mesenchymal stem cells. Nanoscale 2013;5(10):4171-6.

133. Nayak TR, Andersen H, Makam VS, Khaw C, Bae S, Xu X, et al. Graphene for controlled and accelerated osteogenic differentiation of human mesenchymal stem cells. ACS Nano 2011;5(6):4670-8.

134. Akhavan O, Ghaderi E, Shahsavar M. Graphene nanogrids for selective and fast osteogenic differentiation of human mesenchymal stem cells. Carbon 2013;59:200-11.

135. Kim J, Choi KS, Kim Y, Lim KT, Seonwoo H, Park Y, et al. Bioactive effects of graphene oxide cell culture substratum on structure and function of human adipose-derived stem cells. $J$ Biomed Mater Res A 2013;101(12):3520-30.

136. Depan D, Misra R. The interplay between nanostructured carbongrafted chitosan scaffolds and protein adsorption on the cellular 
response of osteoblasts: Structure-function property relationship. Acta Biomater 2013;9(4):6084-94

137. Xie C, Lu X, Han L, Xu J, Wang Z, Jiang L, et al. Biomimetic mineralized hierarchical graphene oxide/chitosan scaffolds with adsorbability for immobilization of nanoparticles for biomedical applications. ACS Appl Mater Interfaces 2016;8(3):1707-17.

138. Nair M, Nancy D, Krishnan AG, Anjusree G, Vadukumpully S, Nair SV. Graphene oxide nanoflakes incorporated gelatin-hydroxyapatite scaffolds enhance osteogenic differentiation of human mesenchymal stem cells. Nanotechnology 2015;26(16):161001.

139. Luo Y, Shen H, Fang Y, Cao Y, Huang J, Zhang M, et al. Enhanced proliferation and osteogenic differentiation of mesenchymal stem cells on graphene oxide-incorporated electrospun poly (lactic-co-glycolic acid) nanofibrous mats. ACS Appl Mater Interfaces 2015;7(11):6331-9.

140. Gu M, Liu Y, Chen T, Du F, Zhao X, Xiong C, et al. Is graphene a promising nano-material for promoting surface modification of implants or scaffold materials in bone tissue engineering? Rev 2014;20(5):477-91.

141. Price RL, Waid MC, Haberstroh KM, Webster TJ. Selective bone cell adhesion on formulations containing carbon nanofibers. Biomaterials 2003;24(11):1877-87.

142. Supronowicz P, Ajayan P, Ullmann K, Arulanandam B, Metzger D, Bizios R. Novel current-conducting composite substrates for exposing osteoblasts to alternating current stimulation. J Biomed Mater Res 2002;59(3):499-506.

143. Shao S, Zhou S, Li L, Li J, Luo C, Wang J, et al. Osteoblast function on electrically conductive electrospun PLA/MWCNTs nanofibers. Biomaterials 2011;32(11):2821-33.

144. Hopley EL, Salmasi S, Kalaskar DM, Seifalian AM. Carbon nanotubes leading the way forward in new generation $3 \mathrm{D}$ tissue engineering. Biotechnol Adv 2014;32(5):1000-14.

145. GhoshMitra S, Diercks DR, Mills NC, Hynds DL, Ghosh S. Role of engineered nanocarriers for axon regeneration and guidance: current status and future trends. Adv Drug Deliv Rev 2012;64(1):110-25.

146. Koch D, Rosoff WJ, Jiang J, Geller HM, Urbach JS. Strength in the periphery: growth cone biomechanics and substrate rigidity response in peripheral and central nervous system neurons. Biophys $J$ 2012;102(3):452-60.

147. Akhavan O, Ghaderi E. The use of graphene in the self-organized differentiation of human neural stem cells into neurons under pulsed laser stimulation. J Mater Chem B 2014;2(34):5602-11.

148. Yang D, Li T, Xu M, Gao F, Yang J, Yang Z, et al. Graphene oxide promotes the differentiation of mouse embryonic stem cells to dopamine neurons. Nanomedicine 2014;9(16):2445-55.

149. Shah S, Yin PT, Uehara TM, Chueng STD, Yang L, Lee KB. Guiding stem cell differentiation into oligodendrocytes using graphenenanofiber hybrid scaffolds. Adv Mater 2014;26(22):3673-80.

150. Yan L, Zhao B, Liu X, Li X, Zeng C, Shi H, et al. Aligned nanofibers from polypyrrole/graphene as electrodes for regeneration of optic nerve via electrical stimulation. ACS Appl Mater Interfaces 2016;8(11):6834-40.

151. Zhou K, Motamed S, Thouas GA, Bernard CC, Li D, Parkington HC, et al. Graphene functionalized scaffolds reduce the inflammatory response and supports endogenous neuroblast migration when implanted in the adult brain. PLoS One 2016;11(3):e0151589.

152. Guo W, Zhang X, Yu X, Wang S, Qiu J, Tang W, et al. Self-powered electrical stimulation for enhancing neural differentiation of mesenchymal stem cells on graphene-poly (3, 4-ethylenedioxythiophene) hybrid microfibers. ACS Nano 2016.

153. Zhang K, Zheng H, Liang S, Gao C. Aligned PLLA nanofibrous scaffolds coated with graphene oxide for promoting neural cell growth. Acta Biomater 2016;37:131-42.

154. Chen C-S, Soni S, Le C, Biasca M, Farr E, Chen EY, et al. Human stem cell neuronal differentiation on silk-carbon nanotube composite. Nanoscale Res Lett 2012;7(1):1.

155. Béduer Al, Seichepine F, Flahaut E, Loubinoux I, Vaysse L, Vieu C. Elucidation of the role of carbon nanotube patterns on the development of cultured neuronal cells. Langmuir 2012;28(50):17363-71.
156. Fabbro A, Sucapane A, Toma FM, Calura E, Rizzetto L, Carrieri C, et al. Adhesion to carbon nanotube conductive scaffolds forces actionpotential appearance in immature rat spinal neurons. PLoS One 2013;8(8):e73621.

157. Yu W, Jiang X, Cai M, Zhao W, Ye D, Zhou Y, et al. A novel electrospun nerve conduit enhanced by carbon nanotubes for peripheral nerve regeneration. Nanotechnology 2014;25(16):165102.

158. Mottaghitalab F, Farokhi M, Zaminy A, Kokabi M, Soleimani M, Mirahmadi F, et al. A biosynthetic nerve guide conduit based on silk/ SWNT/fibronectin nanocomposite for peripheral nerve regeneration. PLoS One 2013;8(9):e74417.

159. Lee JH, Lee J-Y, Yang SH, Lee E-J, Kim H-W. Carbon nanotubecollagen three-dimensional culture of mesenchymal stem cells promotes expression of neural phenotypes and secretion of neurotrophic factors. Acta Biomater 2014;10(10):4425-36.

160. Koppes A, Keating K, McGregor A, Koppes R, Kearns K, Ziemba A, et al. Robust neurite extension following exogenous electrical stimulation within single walled carbon nanotube-composite hydrogels. Acta Biomater 2016;39:34-43.

161. Usmani S, Aurand ER, Medelin M, Fabbro A, Scaini D, Laishram J, et al. 3D meshes of carbon nanotubes guide functional reconnection of segregated spinal explants. Sci Adv 2016;2(7):e1600087.

162. Shadrin I, Khodabukus A, Bursac N. Striated muscle function, regeneration, and repair. Cell Mol Life Sci 2016:1-28.

163. Ku SH, Park CB. Myoblast differentiation on graphene oxide. Biomaterials 2013;34(8):2017-23.

164. Shin YC, Lee JH, Jin L, Kim MJ, Kim Y-J, Hyun JK, et al. Stimulated myoblast differentiation on graphene oxide-impregnated PLGAcollagen hybrid fibre matrices. J Nanobiotechnol 2015;13(1):1.

165. Patel A, Xue Y, Mukundan S, Rohan LC, Sant V, Stolz DB, et al. Cellinstructive graphene-containing nanocomposites induce multinucleated myotube formation. Ann Biomed Eng 2016:1-13.

166. Park J, Park S, Ryu S, Bhang SH, Kim J, Yoon JK, et al. Grapheneregulated cardiomyogenic differentiation process of mesenchymal stem cells by enhancing the expression of extracellular matrix proteins and cell signaling molecules. Adv Healthc Mater 2014;3(2):176-81.

167. Mooney E, Mackle JN, Blond DJ-P, O’Cearbhaill E, Shaw G, Blau WJ, et al. The electrical stimulation of carbon nanotubes to provide a cardiomimetic cue to MSCs. Biomaterials 2012;33(26):6132-9.

168. McKeon-Fischer KD, Rossmeisl JH, Whittington AR, Freeman JW. In vivo skeletal muscle biocompatibility of composite, coaxial electrospun, and microfibrous scaffolds. Tissue Eng Part A 2014;20(13-14):1961-70.

169. Wickham AM, Islam MM, Mondal D, Phopase J, Sadhu V, Tamás É, et al. Polycaprolactone-thiophene-conjugated carbon nanotube meshes as scaffolds for cardiac progenitor cells. Appl Biomater 2014;102(7):1553-61.

170. Patel A, Mukundan S, Wang W, Karumuri A, Sant V, Mukhopadhyay SM, et al. Carbon-based hierarchical scaffolds for myoblast differentiation: Synergy between nano-functionalization and alignment. Acta Biomater 2016;32:77-88.

171. Panczyk T, Wolski P, Lajtar L. Coadsorption of doxorubicin and selected dyes on carbon nanotubes. theoretical investigation of potential application as a pH-controlled drug delivery system. Langmuir 2016;32(19):4719-28.

172. Depan D, Shah J, Misra RDK. Controlled release of drug from folatedecorated and graphene mediated drug delivery system: Synthesis, loading efficiency, and drug release response. Mater Sci Eng C 2011;31(7):1305-12.

173. Singh S, Mehra NK, Jain NK. Development and characterization of the paclitaxel loaded riboflavin and thiamine conjugated carbon nanotubes for cancer treatment. Pharm Res 2016;33(7):1769-81.

174. Gao P, Liu MY, Tian JW, Deng FJ, Wang K, Xu DZ, et al. Improving the drug delivery characteristics of graphene oxide based polymer nanocomposites through the "one-pot" synthetic approach of singleelectron-transfer living radical polymerization. Appl Surf Sci 2016;378:22-9. 
175. Fedeli S, Brandi A, Venturini L, Chiarugi P, Giannoni E, Paoli P, et al. The "click-on-tube" approach for the production of efficient drug carriers based on oxidized multi-walled carbon nanotubes. $J$ Mater Chem B 2016;4(21):3823-31.

176. Cao LL, Liang YB, Zhao FJ, Zhao XJ, Chen ZC. Chelerythrine and $\mathrm{Fe} 3 \mathrm{O} 4$ loaded multi-walled carbon nanotubes for targeted cancer therapy. J Biomed Nanotechnol 2016;12(6):1312-22.

177. Liu KP, Wang YM, Li HM, Duan YX. A facile one-pot synthesis of starch functionalized graphene as nano-carrier for $\mathrm{pH}$ sensitive and starch-mediated drug delivery. Biointerfaces 2015;128:86-93.

178. Han S, Kwon T, Um JE, Haam S, Kim WJ. Highly selective photothermal therapy by a phenoxylated-dextran-functionalized smart carbon nanotube platform. Adv Healthc Mater 2016;5(10):1147-56.

179. Sharker SM, Lee JE, Kim SH, Jeong JH, In I, Lee H, et al. pH triggered in vivo photothermal therapy and fluorescence nanoplatform of cancer based on responsive polymer-indocyanine green integrated reduced graphene oxide. Biomaterials 2015;61:229-38.

180. Battogtokh G, Ko YT. Graphene oxide-incorporated pH-responsive folate-albumin-photosensitizer nanocomplex as image-guided dual therapeutics. J Control Release 2016;234:10-20.

181. De Souza LA, Nogueira CAS, Ortega PFR, Lopes JF, Calado HDR, Lavall RL, et al. Inclusion complex between cisplatin and single-walled carbon nanotube: An integrated experimental and theoretical approach. Inorg Chim Acta 2016;447:38-44.

182. Anderson T, Hu R, Yang C, Yoon HS, Yong K-T. Pancreatic cancer gene therapy using an siRNA-functionalized single walled carbon nanotubes (SWNTs) nanoplex. Biomater Sci 2014;2(9):1244.

183. Siu KS, Zheng X, Liu Y, Zhang Y, Zhang X, Chen D, et al. Singlewalled carbon nanotubes noncovalently functionalized with lipid modified polyethylenimine for siRNA delivery in vitro and in vivo. Bioconjug Chem 2014;25(10):1744-51.

184. Weaver CL, LaRosa JM, Luo XL, Cui XT. Electrically controlled drug delivery from graphene oxide nanocomposite films. ACS Nano 2014;8(2):1834-43.

185. Mo R, Jiang TY, Sun WJ, Gu Z. ATP-responsive DNA-graphene hybrid nanoaggregates for anticancer drug delivery. Biomaterials 2015;50:67-74.

186. de Sousa IP, Buttenhauser K, Suchaoin W, Partenhauser A, Perrone M, Matuszczak B, et al. Thiolated graphene oxide as promising mucoadhesive carrier for hydrophobic drugs. Pharm 2016;509(1-2):360-7.

187. Singh S, Dubey VK. Multiwalled carbon nanotube-superoxide dismutase conjugate towards alleviating induced oxidative stress. Pept Res Ther 2016;22(2):171-7.
188. Shi H, Wei J, Qiang L, Chen X, Meng X. Fluorescent carbon dots for bioimaging and biosensing applications. J Biomed Nanotechnol 2014;10(10):2677-99.

189. Wang D, Chen J-F, Dai L. Recent advances in graphene quantum dots for fluorescence bioimaging from cells through tissues to animals. Part Part Syst Charact 2015;32(5):515-23.

190. Ag D, Seleci M, Bongartz R, Can M, Yurteri S, Cianga I, et al. From invisible structures of SWCNTs toward fluorescent and targeting architectures for cell imaging. Biomacromolecules 2013;14(10):3532-41.

191. Hu ZY, Pantos GD, Kuganathan N, Arrowsmith RL, Jacobs RMJ, KociokKohn G, et al. Interactions between amino acid-tagged naphthalenediimide and single walled carbon nanotubes for the design and construction of new bioimaging probes. Adv Funct Mater 2012;22(3):503-18.

192. Khandare JJ, Jalota-Badhwar A, Satavalekar SD, Bhansali SG, Aher ND, Kharas F, et al. PEG-conjugated highly dispersive multifunctional magnetic multi-walled carbon nanotubes for cellular imaging. Nanoscale 2012;4(3):837-44.

193. Kundu A, Nandi S, Das P, Nandi AK. Fluorescent graphene oxide via polymer grafting: An efficient nanocarrier for both hydrophilic and hydrophobic drugs. ACS Appl Mater Interfaces 2015;7(6):3512-23.

194. Liu X, Marangon I, Melinte G, Wilhelm C, Menard-Moyon C, Pichon $\mathrm{BP}$, et al. Design of covalently functionalized carbon nanotubes filled with metal oxide nanoparticles for imaging, therapy, and magnetic manipulation. ACS Nano 2014;8(11):11290-304.

195. Shi X, Gong H, Li Y, Wang C, Cheng L, Liu Z. Graphene-based magnetic plasmonic nanocomposite for dual bioimaging and photothermal therapy. Biomaterials 2013;34(20):4786-93.

196. Wate PS, Banerjee SS, Jalota-Badhwar A, Mascarenhas RR, Zope KR, Khandare J, et al. Cellular imaging using biocompatible dendrimerfunctionalized graphene oxide-based fluorescent probe anchored with magnetic nanoparticles. Nanotechnology 2012:23(41).

197. Tian JW, Luo YP, Huang LW, Feng YQ, Ju HX, Yu BY. Pegylated folate and peptide-decorated graphene oxide nanovehicle for in vivo targeted delivery of anticancer drugs and therapeutic self-monitoring. Biosens Bioelectron 2016;80:519-24.

198. Dong H, Gao W, Yan F, Ji H, Ju H. Fluorescence resonance energy transfer between quantum dots and graphene oxide for sensing biomolecules. Anal Chem 2010;82(13):5511-7.

199. Lu CH, Yang HH, Zhu CL, Chen X, Chen GN. A graphene platform for sensing biomolecules. Angew Chem Int Ed Engl 2009;48(26):4785-7.

200. Barsan MM, Pifferi V, Falciola L, Brett CMA. New CNT/poly(brilliant green) and CNT/poly(3,4-ethylenedioxythiophene) based electrochemical enzyme biosensors. Anal Chim Acta 2016;927:35-45. 